\title{
МЕТОД РОЗРАХУНКУ ВІЛЬНОЇ ЕНЕРГІї ТРИВИМІРНОЇ ІЗИНГОПОДІБНОЇ СИСТЕМИ З ВРАХУВАННЯМ ПОПРАВКИ НА УСЕРЕДНЕННЯ ПОТЕНЦІАЛУ ВЗАЕМОДІЇ
}

\author{
І.Р. ЮХновСьКИй, М.П. КОзловсьКий, І.В. Пилюк
}

УдК 538.9; 537.611.2 (c) 2012

Інститут фізики конденсованих систем НАН України

(Вул. Свенціцъкого, 1, Лъвів 79011; е-mail: piv@істр. lviv. иа)

Роботу присвячено теоретичному вивченню критичної поведінки тривимірних модельних систем. Виконано розрахунок статистичної суми та вільної енергії однокомпонентної спінової системи з використанням негаусового розподілу флуктуацій параметра порядку. Особливістю запропонованого методу розрахунку є врахування залежності фур'є-образу потенціалу взаємодії від хвильового вектора. Це приводить до відмінного від нуля критичного показника кореляційної функції $\eta$ та перенормування значень інших критичних показників (кореляційної довжини, сприйнятливості тощо). Розрахунок останніх здійснено з використанням методу ренормалізаційної групи на основі отриманих у роботі рекурентних співвідношень між коефіцієнтами розподілів флуктуацій у суміжних блочних структурах.

\section{1. Вступ}

Основною задачею фізики фазових переходів є опис співвідношень між макроскопічними, експериментально вимірювальними величинами, виходячи з мікроскопічних параметрів системи і сил, що діють між частинками, з яких вона складається. Основи мікроскопічного підходу теорії фазових переходів було закладено в роботах $[1,2]$, де суттєвим чином використано ідеї, що випливають з гіпотези подібності $[3,4]$. Свій подальший розвиток мікроскопічна теорія фазових переходів отримала в методі колективних змінних (K3), який в [5-7] був узагальнений на випадок спінових систем. Для однокомпонентної спінової моделі вдалось отримати не лише значення критичних показників теплоємності, середнього спінового моменту, сприйнятливості та інших характеристик, а й знайти i дослідити поблизу температури фазового переходу $T_{c}$ явні вирази для цих характеристик як функції температури, магнітного поля та мікроскопічних параметрів гамільтоніана. 3 методом КЗ та одержаними на його основі деякими результатами досліджень можна ознайомитись в [8-12].

Теоретичний опис критичної поведінки реальних систем на певному етапі розрахунку зводиться до опису фазового переходу в деякій моделі. Метод КЗ, основи якого стосовно тривимірної моделі Ізинга - базової моделі фазового переходу - закладені в 70-80-х роках минулого століття, постійно вдосконалювався. Варто зауважити, що модель Ізинга, незважаючи на свою простоту, має, з одного боку, широку область реалістичних застосувань, а 3 іншого боку - може розглядатися як модель, що служить своєрідним еталоном при дослідженні багатьох інших моделей, значно складніших за своєю побудовою. Розвиток методу розрахунку основних термодинамічних та структурних характеристик базової моделі фазового переходу відкриває шлях до опису складніших фізичних систем. Саме тому максимально повний розв'язок тривимірної ізингоподібної системи є ключем до опису критичної поведінки багатьох фізичних об'єктів. Одновісні магнетики, прості рідини, бінарні сплави, міцелярні системи - це далеко неповний перелік об'єктів, яким притаманна ізингоподібна поведінка. Критична поведінка класу універсальності тривимірної моделі Ізинга виявлена в системах з сильними та електрослабкими взаємодіями, що спостерігаються у фізиці високих енергій.

У методі К3 фазовий простір КЗ $\rho_{\mathbf{k}}$ при розрахунку статистичної суми тривимірної ізингоподібної 
системи розбивається на шари $\left\{\rho_{\mathbf{k}}\right\}_{B_{n+1}, B_{n}}$, які відповідають певним значенням хвильового вектора $\mathbf{k}$ $\left(B_{n+1}=B_{n} / s, s>1\right.$ - параметр поділу). Розрахунок статистичної суми ведеться поетапно, починаючи $з$ шарів фазового простору К3, що відповідають змінним $\rho_{\mathbf{k}}$ з великими значеннями хвильового вектоpa $\mathbf{k}$, і закінчуючи $\rho_{\mathbf{k}}$ з $k \rightarrow 0$. При цьому в кожному з шарів фур'є-образ потенціалу взаємодії замінюється його середнім значенням. Останнє приводить до нульового значення критичного показника $\eta$, який характеризує поведінку парної кореляційної функції при $T=T_{c}$.

Обчислення так званого малого критичного показника $\eta$ - нетривіальний процес (див., наприклад, дослідження в рамках непертурбативного ренормгрупового (РГ) підходу $[13,14]$, подібного до методу К3). $\mathrm{У}$ даній роботі ми розрахували термодинамічні характеристики поблизу $T_{c}$ з врахуванням поправки на усереднення фур'є-образу потенціалу, яка веде до ненульового значення показника $\eta$ і в попередніх дослідженнях (зокрема в [15-18]) не бралась до уваги. Запропонована у даній статті на основі підходу КЗ методика розрахунку термодинаміки системи завдяки врахуванню як критичного показника кореляційної функції, так і змін (порівняно з випадком $\eta=0$ ) для координат фіксованої точки, рекурентних співвідношень (РC) і самих коефіцієнтів термодинамічних характеристик, набуває більш загального і повного характеру.

\section{2. Постановка задачі}

Розглядається система $N$ взаємодіючих частинок, розміщених у вузлах простої кубічної гратки з періодом $c$. Стан кожної частинки визначається власним значенням оператора $z$-компоненти її спіна, яке може бути рівним або +1 (спін орієнтований вверх), або -1 (вниз). Гамільтоніан системи записується у вигляді

$H=-\frac{1}{2} \sum_{\mathbf{i}, \mathbf{j}} \Phi\left(r_{\mathbf{i} \mathbf{j}}\right) s_{\mathbf{i}} s_{\mathbf{j}}-h \sum_{\mathbf{i}} s_{\mathbf{i}}$,

де $s_{\mathbf{i}}$ - оператор $z$-компоненти спіна в $\mathbf{i}$-му вузлі, $\Phi\left(r_{\mathbf{i j}}\right)$ - потенціал взаємодії, який представляється експоненційно спадною функцією

$\Phi\left(r_{\mathbf{i j}}\right)=A \exp \left(-r_{\mathbf{i j}} / b\right)$

Тут $A$ - стала, $r_{\mathbf{i j}}$ - відстань між $\mathbf{i}$-м та $\mathbf{j}$-м вузлом гратки, $b$ - радіус ефективної взаємодії. Статистична сума моделі в представленні $\mathrm{K} 3 \rho_{\mathbf{k}}$ за відсутності зовнішнього магнітного поля $\mathcal{H}$ (або при $h=\mu_{\mathrm{B}} \mathcal{H}=0$, де $\mu_{\mathrm{B}}$ - магнетон Бора) записується у вигляді

$$
\begin{aligned}
& Z=2^{N} 2^{\left(N^{\prime}-1\right) / 2} e^{a_{0}^{\prime} N^{\prime}} \int \exp \left\{-\frac{1}{2} \sum_{k \leq B^{\prime}} d^{\prime}(k) \rho_{\mathbf{k}} \rho_{-\mathbf{k}}-\right. \\
& \left.-\frac{1}{4 !}\left(N^{\prime}\right)^{-1} \sum_{k_{1}, \ldots, k_{4} \leq B^{\prime}} a_{4}^{\prime} \rho_{\mathbf{k}_{1}} \cdots \rho_{\mathbf{k}_{4}} \delta_{\mathbf{k}_{1}+\cdots+\mathbf{k}_{4}}\right\}(d \rho)^{N^{\prime}} .
\end{aligned}
$$

Тут використано наближення четвірної базисної густини міри $[8,19], B^{\prime}=(b \sqrt{2})^{-1}, N^{\prime}=N s_{0}^{-3}\left(s_{0}=\right.$ $B / B^{\prime}, B=\pi / c-$ границя півзони Бріллюена), а для $d^{\prime}(k)$ маємо

$d^{\prime}(k)=a_{2}^{\prime}-\beta \tilde{\Phi}(k)$.

Величина $\beta=1 /(k T)$ - обернена температура. Для фур'є-образу потенціалу взаємодії (2) використовується параболічна апроксимація

$\tilde{\Phi}(k)= \begin{cases}\tilde{\Phi}(0)\left(1-2 b^{2} k^{2}\right), & k \leq B^{\prime}, \\ 0, & B^{\prime}<k \leq B,\end{cases}$

яка по суті не впливає на методику опису подій поблизу точки фазового переходу. Основний внесок у термодинамічні і структурні функції походить від області хвильових векторів при $k \rightarrow 0$. Інтервал великих значень $\mathbf{k}$ формуе лише початкові значення коефіцієнтів $a_{0}^{\prime}, a_{2}^{\prime}$ і $a_{4}^{\prime}$, які не впливають на значення критичних показників. В наближенні (5) маємо

$a_{0}^{\prime}=\ln \left[\frac{1}{\sqrt{2 \pi}}\left(y^{\prime}\right)^{1 / 2} e^{\left(y^{\prime}\right)^{2} / 4} U\left(0, y^{\prime}\right)\right]$,

$a_{2}^{\prime}=y^{\prime} U\left(y^{\prime}\right), \quad a_{4}^{\prime}=\left(y^{\prime}\right)^{2} \varphi\left(y^{\prime}\right)$,

де $y^{\prime}=2^{1 / 4} \sqrt{3} \pi^{3 / 2}(b / c)^{3 / 2}$, а функції $U\left(y^{\prime}\right)$ і $\varphi\left(y^{\prime}\right)$ виражаються через функції параболічного циліндра (функції Вебера) $U\left(a, y^{\prime}\right)[19]$ :

$U\left(y^{\prime}\right)=U\left(1, y^{\prime}\right) / U\left(0, y^{\prime}\right)$

$\varphi\left(y^{\prime}\right)=3 U^{2}\left(y^{\prime}\right)+2 y^{\prime} U\left(y^{\prime}\right)-2$.

Коефіцієнти $a_{2 l}^{\prime}$ залежать від відношення радіуса дії потенціалу $b$ до сталої гратки $c$. У випадку $b=c$ отримуємо

$a_{0}^{\prime}=-0,921747, \quad a_{2}^{\prime}=0,988929, \quad a_{4}^{\prime}=0,021120$.

Розрахунок виразу для статистичної суми (3) виконується шляхом "пошарового" інтегрування (див., наприклад, $[7,8,11])$. У кожному $n$-му шарі K3 $\rho_{\mathbf{k}}$, якому відповідає область хвильових векторів $B_{n+1}<$ $k \leq B_{n}$, здійснюється заміна фур'є-образу $\tilde{\Phi}(k)$ на середне значення

$\tilde{\Phi}\left(B_{n+1}, B_{n}\right)=\tilde{\Phi}(0)-2 \tilde{\Phi}(0) b^{2} B_{n}^{2} \bar{q}$. 
Тут $B_{n}=B^{\prime} s^{-n}$, а величина $\bar{q}$ відповідає середньому значенню $k^{2}$ на інтервалі $(1 / s, 1]$. При геометричному способі усереднення, який використовується в даних обчисленнях, маємо $\bar{q}=3\left(1-s^{-5}\right) /\left[5\left(1-s^{-3}\right)\right]$. РС, що виникають в процесі інтегрування, набувають вигляду $[7,19]$

$d_{n+1}\left(B_{n+2}, B_{n+1}\right)=d_{n}\left(B_{n+1}, B_{n}\right) N\left(x_{n}\right)-\Delta_{n}$,

$a_{4}^{(n+1)}=a_{4}^{(n)} s^{-3} E\left(x_{n}\right)$,

де $d_{n}\left(B_{n+1}, B_{n}\right)=a_{2}^{(n)}-\beta \tilde{\Phi}\left(B_{n+1}, B_{n}\right), \Delta_{n}=\beta \tilde{\Phi}(0) \times$ $\times s^{-2 n}\left(1-s^{-2}\right) \bar{q}$, а для функцій $N\left(x_{n}\right), E\left(x_{n}\right)$ маємо

$N\left(x_{n}\right)=\frac{y_{n} U\left(y_{n}\right)}{x_{n} U\left(x_{n}\right)}, \quad E\left(x_{n}\right)=s^{6} \frac{\varphi\left(y_{n}\right)}{\varphi\left(x_{n}\right)}$.

Аргументи $x_{n}$ та $y_{n}$, що входять до складу (10), визначаються виразами

$x_{n}=\sqrt{3} d_{n}\left(B_{n+1}, B_{n}\right)\left(a_{4}^{(n)}\right)^{-1 / 2}$,

$y_{n}=s^{3 / 2} U\left(x_{n}\right)\left(3 / \varphi\left(x_{n}\right)\right)^{1 / 2}$.

В окремому випадку (при $x_{n} \gg 1$ ) загальні РC (9) зводяться до РС К. Вільсона [20,21]. Використання РC (9) дає змогу досліджувати властивості тривимірних систем у критичній області.

Тепер при розрахунку статистичної суми системи будемо брати до уваги величину

$\Delta \tilde{\Phi}(k)=q-2 b^{2} \beta \tilde{\Phi}(0) k^{2}$

яка відповідає відхиленню $\beta \tilde{\Phi}(k)$ від середнього значення $\beta \tilde{\Phi}\left(B_{1}, B^{\prime}\right)$, тобто враховуватимемо поправку на усереднення $\tilde{\Phi}(k)$. Тут $q=\bar{q} \beta \tilde{\Phi}(0)$. Вважаючи в подальшому величину $\Delta \tilde{\Phi}(k)$ малою поправкою до відповідного середнього значення, дослідимо їі вплив на критичні властивості системи та розвинемо метод розрахунку вільної енергії системи з врахуванням залежності фур'є-образу потенціалу $\tilde{\Phi}(k)$ від хвильового вектора.

\section{3. Розрахунок статистичної суми системи}

Результат інтегрування статистичної суми за K3 $\rho_{\mathbf{k}}$, індекси $\mathbf{k}$ яких потрапляють в інтервал $B_{1}<k \leq B^{\prime}$ $\left(B_{1}=B^{\prime} / s\right)$, представляється у вигляді $[8,10,22]$

$Z=2^{N} 2^{\left(N_{1}-1\right) / 2} Q_{0}[Q(P)]^{N_{1}} \times$

$\times \int \exp \left\{-\frac{1}{2} \sum_{k \leq B_{1}}\left[d^{\prime}(k)-d^{\prime}\left(B_{1}, B^{\prime}\right)\right] \rho_{\mathbf{k}} \rho_{-\mathbf{k}}\right\} \times$
$\times\left(1+\hat{\Delta}_{g}+\cdots\right) \exp \left[-\frac{1}{2} R_{2} \sum_{k \leq B_{1}} \rho_{\mathbf{k}} \rho_{-\mathbf{k}}-\frac{1}{4 !}\left(N_{1}\right)^{-1} \times\right.$

$\left.\times R_{4} \sum_{k_{1}, \ldots, k_{4} \leq B_{1}} \rho_{\mathbf{k}_{1}} \cdots \rho_{\mathbf{k}_{4}} \delta_{\mathbf{k}_{1}+\cdots+\mathbf{k}_{4}}\right](d \rho)^{N_{1}}$,

де $N_{1}=N^{\prime} s^{-3}, Q_{0}=\left[e^{a_{0}^{\prime}} Q(d)\right]^{N^{\prime}}$ - парціальна статистична сума нульового шару фазового простору K3 [19],

$Q(d)=(2 \pi)^{1 / 2}\left(3 / a_{4}^{\prime}\right)^{1 / 4} e^{x^{2} / 4} U(0, x)$,

$Q(P)=(2 \pi)^{-1 / 2} s^{3 / 4}\left(a_{4}^{\prime} / \varphi(x)\right)^{1 / 4} e^{y^{2} / 4} U(0, y)$,

$x=x_{n=0}=\sqrt{3} d^{\prime}\left(B_{1}, B^{\prime}\right)\left(a_{4}^{\prime}\right)^{-1 / 2}$,

$y=s^{3 / 2} U(x)(3 / \varphi(x))^{1 / 2}$.

Для $R_{2}$ і $R_{4}$ маємо [22]

$R_{2}=d^{\prime}\left(B_{1}, B^{\prime}\right) N(x), \quad R_{4}=a_{4}^{\prime} s^{-3} E(x)$.

Функції $N(x), E(x)$ означені в (10). Оператор $\hat{\Delta}_{g}$ задається виразом $[11,22]$

$\hat{\Delta}_{g}=\frac{1}{2(2 \pi i)^{2}} \sum_{\mathbf{l}_{1}, \mathbf{l}_{2}}\left(N^{\prime}\right)^{-1} \sum_{B_{1}<k \leq B^{\prime}} \Delta g(k) e^{-i \mathbf{k}\left(\mathbf{l}_{1}-\mathbf{l}_{2}\right)} \times$

$\times\left\{\left(\frac{S_{4}}{3 !}\right)^{2} \frac{1}{(2 \pi i)^{6}}\left(N^{\prime}\right)^{-3} \sum_{k_{1}, \ldots, k_{6} \leq B_{1}} \frac{\partial^{6}}{\partial \rho_{\mathbf{k}_{1}} \cdots \partial \rho_{\mathbf{k}_{6}}} \times\right.$

$\times \exp \left[-i\left(\mathbf{k}_{1}+\mathbf{k}_{2}+\mathbf{k}_{3}\right) \mathbf{l}_{1}-i\left(\mathbf{k}_{4}+\mathbf{k}_{5}+\mathbf{k}_{6}\right) \mathbf{l}_{2}\right]+$

$+2 \frac{S_{4} S_{6}}{3 ! 5 !} \frac{1}{(2 \pi i)^{8}}\left(N^{\prime}\right)^{-4} \sum_{k_{1}, \ldots, k_{8} \leq B_{1}} \frac{\partial^{8}}{\partial \rho_{\mathbf{k}_{1}} \cdots \partial \rho_{\mathbf{k}_{8}}} \times$

$\times \exp \left[-i\left(\mathbf{k}_{1}+\mathbf{k}_{2}+\mathbf{k}_{3}\right) \mathbf{l}_{1}-i\left(\mathbf{k}_{4}+\cdots+\mathbf{k}_{8}\right) \mathbf{l}_{2}\right]+$

$+\frac{1}{(2 \pi i)^{10}}\left(N^{\prime}\right)^{-5} \sum_{k_{1}, \ldots, k_{10} \leq B_{1}} \frac{\partial^{10}}{\partial \rho_{\mathbf{k}_{1}} \cdots \partial \rho_{\mathbf{k}_{10}}}\left[2 \frac{S_{4} S_{8}}{3 ! 7 !} \times\right.$

$\times \exp \left[-i\left(\mathbf{k}_{1}+\mathbf{k}_{2}+\mathbf{k}_{3}\right) \mathbf{l}_{1}-i\left(\mathbf{k}_{4}+\cdots+\mathbf{k}_{10}\right) \mathbf{l}_{2}\right]+$

$+\left(\frac{S_{6}}{5 !}\right)^{2} \exp \left[-i\left(\mathbf{k}_{1}+\cdots+\mathbf{k}_{5}\right) \mathbf{l}_{1}-\right.$

$\left.\left.-i\left(\mathbf{k}_{6}+\cdots+\mathbf{k}_{10}\right) \mathbf{l}_{2}\right]\right]+2 \frac{S_{6} S_{8}}{5 ! 7 !} \frac{1}{(2 \pi i)^{12}}\left(N^{\prime}\right)^{-6} \times$

$\times \sum_{k_{1}, \ldots, k_{12} \leq B_{1}} \frac{\partial^{12}}{\partial \rho_{\mathbf{k}_{1}} \cdots \partial \rho_{\mathbf{k}_{12}}} \exp \left[-i\left(\mathbf{k}_{1}+\cdots+\mathbf{k}_{5}\right) \mathbf{l}_{1}-\right.$

$\left.-i\left(\mathbf{k}_{6}+\cdots+\mathbf{k}_{12}\right) \mathbf{l}_{2}\right]+\left(\frac{S_{8}}{7 !}\right)^{2} \frac{1}{(2 \pi i)^{14}}\left(N^{\prime}\right)^{-7} \times$

$\times \sum_{k_{1}, \ldots, k_{14} \leq B_{1}} \frac{\partial^{14}}{\partial \rho_{\mathbf{k}_{1}} \cdots \partial \rho_{\mathbf{k}_{14}}} \exp \left[-i\left(\mathbf{k}_{1}+\cdots+\mathbf{k}_{7}\right) \mathbf{l}_{1}-\right.$

$\left.\left.-i\left(\mathbf{k}_{8}+\cdots+\mathbf{k}_{14}\right) \mathbf{l}_{2}\right]+\cdots\right\}$. 
Тут

$S_{2}=(2 \pi)^{2}\left(\frac{3}{a_{4}^{\prime}}\right)^{1 / 2} U(x), \quad S_{4}=(2 \pi)^{4} \frac{3}{a_{4}^{\prime}} \varphi(x)$,

$S_{6}=(2 \pi)^{6}\left(\frac{3}{a_{4}^{\prime}}\right)^{3 / 2} \varphi_{1}(x), \quad S_{8}=(2 \pi)^{8}\left(\frac{3}{a_{4}^{\prime}}\right)^{2} \varphi_{2}(x)$,

а функції $\varphi_{l}(x)$ визначаються співвідношеннями

$\varphi(x)=3 U^{2}(x)+2 x U(x)-2$,

$\varphi_{1}(x)=30 U^{3}(x)+30 x U^{2}(x)+4\left(x^{2}-6\right) U(x)-4 x$,

$\varphi_{2}(x)=630 U^{4}(x)+840 x U^{3}(x)+84\left(3 x^{2}-8\right) U^{2}(x)+$

$+8 x\left(x^{2}-45\right) U(x)-8 x^{2}+120$.

Підсумовування за вузлами $\mathbf{l}_{1}, \mathbf{l}_{2}$ в (16) здійснюється для гратки з періодом $c^{\prime}=\pi b \sqrt{2}$, значення хвильового вектора змінюються в інтервалі $\left[0, B^{\prime}\right]$. Роль $\Delta g(k)$ відіграє величина

$\Delta g(k)=\frac{\Delta \tilde{\Phi}(k)}{1-S_{2}(2 \pi)^{-2} \Delta \tilde{\Phi}(k)}$.

Оператор $\hat{\Delta}_{g}$ в (13) діє на функцію

$I\left(R_{2}, R_{4}\right)=\exp \left[-\frac{1}{2} R_{2} \sum_{k \leq B_{1}} \rho_{\mathbf{k}} \rho_{-\mathbf{k}}-\frac{1}{4 !}\left(N_{1}\right)^{-1} \times\right.$

$\left.\times R_{4} \sum_{k_{1}, \ldots, k_{4} \leq B_{1}} \rho_{\mathbf{k}_{1}} \cdots \rho_{\mathbf{k}_{4}} \delta_{\mathbf{k}_{1}+\cdots+\mathbf{k}_{4}}\right]$,

для якої в подальшому будемо обмежуватись врахуванням першого доданка в експоненті. Ця обставина зв'язана $з$ малою величиною внеску від $R_{4}$ в порівнянні з внеском від $R_{2}$ (числові оцінки відношення $R_{4} /\left(6 R_{2}^{2}\right)$ для різних значень параметра РГ $s$ наведено в роботі [22] і мають порядок $\left.10^{-4}\right)$.

Врахування поправки, що вноситься оператором $\hat{\Delta}_{g}$, розглядається в лінійному наближенні за $\Delta \tilde{\Phi}(k)$. При цьому у виразі (16) будемо обмежуватись першим доданком, пропорційним до $\frac{\partial^{6}}{\partial \rho_{\mathbf{k}_{1}} \cdots \partial \rho_{\mathbf{k}_{6}}}$. У вказаному наближенні маємо

$\hat{\Delta}_{g}^{(1)}=\frac{1}{2} \sum_{k_{1}, \ldots, k_{6} \leq B_{1}}\left(\frac{\varphi(x)}{2 a_{4}^{\prime}}\right)^{2} \frac{\partial^{6}}{\partial \rho_{\mathbf{k}_{1}} \cdots \partial \rho_{\mathbf{k}_{6}}}\left(N^{\prime}\right)^{-4} \times$

$\times \sum_{B_{1}<k \leq B^{\prime}} \Delta g(k) \sum_{\mathbf{l}_{1}, \mathbf{l}_{2}} \exp \left[-i\left(\mathbf{k}_{1}+\mathbf{k}_{2}+\mathbf{k}_{3}+\mathbf{k}\right) \mathbf{l}_{1}-\right.$

$\left.-i\left(\mathbf{k}_{4}+\mathbf{k}_{5}+\mathbf{k}_{6}-\mathbf{k}\right) \mathbf{l}_{2}\right]$.

Коротке зауваження щодо прийняття до уваги в (16) інших доданків буде наведено нижче.
Дія оператора (20) на функцію (19) приводить до результату

$$
\begin{aligned}
& \Delta_{g}^{(1)}=-\frac{3}{4}\left(\frac{\varphi(x)}{a_{4}^{\prime}}\right)^{2} R_{2}^{3} \sum_{\mathbf{l}} \mathcal{I}_{1}(\bar{x}) \mathcal{I}_{2}(\bar{x}) \times \\
& \times\left[N^{\prime} \mathcal{I}_{1}^{2}(\bar{x})-3 R_{2} \mathcal{I}_{1}(\bar{x}) \sum_{k \leq B_{1}} \rho_{\mathbf{k}} \rho_{-\mathbf{k}} e^{-i \mathbf{k} \mathbf{l}}+\frac{3}{2} R_{2}^{2} s^{-3} \times\right. \\
& \left.\times\left(N_{1}\right)^{-1} \sum_{k_{1}, \ldots, k_{4} \leq B_{1}} \rho_{\mathbf{k}_{1}} \cdots \rho_{\mathbf{k}_{4}} e^{-i\left(\mathbf{k}_{1}+\mathbf{k}_{3}\right) \mathbf{l}} \delta_{\mathbf{k}_{1}+\cdots+\mathbf{k}_{4}}\right] \cdot(21)
\end{aligned}
$$

Тут $\mathbf{l}=\mathbf{l}_{1}-\mathbf{l}_{2}, \bar{x}=l / c^{\prime}$. Величини $\mathcal{I}_{1}(\bar{x}), \mathcal{I}_{2}(\bar{x})$ обчислюються за допомогою переходу до сферичної зони Бріллюена та інтегрування за $k \in\left[0, B_{1}\right]$ :

$\mathcal{I}_{1}(\bar{x})=\frac{1}{N^{\prime}} \sum_{k \leq B_{1}} e^{-i \mathbf{k} \mathbf{l}}=\delta_{\mathbf{l}}-Y(\bar{x})$,
$\mathcal{I}_{2}(\bar{x})=\frac{1}{N^{\prime}} \sum_{B_{1}<k \leq B^{\prime}} \Delta g(k) e^{-i \mathbf{k} \mathbf{l}}=q t(Y(\bar{x})-\mathcal{Z}(\bar{x}))$.

Для функцій $Y(\bar{x})$ та $\mathcal{Z}(\bar{x})$, що входять в $(22)$, отримуємо

$Y(\bar{x})=\frac{1}{N^{\prime}} \sum_{B_{1}<k \leq B^{\prime}} e^{-i \mathbf{k} \mathbf{l}}=\frac{3}{(\pi \bar{x})^{3}}[\sin (\pi \bar{x})-$

$\left.-\pi \bar{x} \cos (\pi \bar{x})-\sin \left(\frac{\pi \bar{x}}{s}\right)+\frac{\pi \bar{x}}{s} \cos \left(\frac{\pi \bar{x}}{s}\right)\right]$

$\mathcal{Z}(\bar{x})=\frac{2 \beta \tilde{\Phi}(0) b^{2}}{q} \frac{1}{N^{\prime}} \sum_{B_{1}<k \leq B^{\prime}} k^{2} e^{-i \mathbf{k} \mathbf{l}}=$

$=\frac{3}{\pi^{2} \bar{q} \bar{x}^{2}}\left\{3 \frac{\sin (\pi \bar{x})}{\pi \bar{x}}-\cos (\pi \bar{x})+6\left(\frac{\cos (\pi \bar{x})}{(\pi \bar{x})^{2}}-\right.\right.$

$\left.-\frac{\sin (\pi \bar{x})}{(\pi \bar{x})^{3}}\right)-s^{-3}\left[3 \frac{\sin (\pi \bar{x} / s)}{\pi \bar{x} / s}-\cos (\pi \bar{x} / s)+\right.$

$\left.\left.+6\left(\frac{\cos (\pi \bar{x} / s)}{(\pi \bar{x} / s)^{2}}-\frac{\sin (\pi \bar{x} / s)}{(\pi \bar{x} / s)^{3}}\right)\right]\right\}$.

Величина

$t=\left\langle\frac{1}{1-\sqrt{3 / a_{4}^{\prime}} U(x) \Delta \tilde{\Phi}(k)}\right\rangle_{B_{1}, B^{\prime}}$,

де символ $\langle\cdots\rangle_{B_{1}, B^{\prime}}$ означає геометричне усереднення на інтервалі $\left(B_{1}, B^{\prime}\right]$, визначається згідно з фор-

ISSN 2071-0194. Укр. фіз. журн. 2012. T. 57, №1 
мулами

$t=\sqrt{\frac{\bar{a}_{4}^{\prime}}{3}} \frac{1}{U(x)} t_{0}, \quad t_{0}=\frac{3}{1+s^{-1}+s^{-2}}+\frac{3 a}{1-s^{-3}} I_{0}$,

$\bar{a}_{4}^{\prime}=a_{4}^{\prime}(\beta \tilde{\Phi}(0))^{-2}, \quad a=\bar{q}-\sqrt{\frac{\bar{a}_{4}^{\prime}}{3}} \frac{1}{U(x)}$,

$I_{0}= \begin{cases}\frac{1}{2 \sqrt{a}} \ln \left|\frac{(1-\sqrt{a})\left(s^{-1}+\sqrt{a}\right)}{(1+\sqrt{a})\left(s^{-1}-\sqrt{a}\right)}\right|, & a>0, \\ \frac{1}{\sqrt{|a|}}\left(\operatorname{arctg} \frac{1}{\sqrt{|a|}}-\operatorname{arctg} \frac{1}{\sqrt{|a|}}\right), & a<0 .\end{cases}$

Зазначимо, що виділення символу Кронекера $\delta_{\mathbf{l}}$ у виразі для $\mathcal{I}_{1}(\bar{x})$ (див. (22)) запропоноване в [8] i $є$ важливим елементом розрахунків. Така процедура дає змогу виділити із області хвильових векторів $k \in\left[0, B^{\prime}\right]$ ту їі частину $k \in\left(B_{1}, B^{\prime}\right]$, де саме й враховується поправка на усереднення потенціалу. Відсутність цієї процедури в [23] привела до першої невдалої спроби розрахунку критичного показника $\eta$ в методі КЗ. У випадку $s \rightarrow 1$ маємо $Y(\bar{x}) \rightarrow 0, B_{1} \rightarrow B^{\prime}$ і величина $\mathcal{I}_{1}(\bar{x})$ переходить у символ Кронекера $\delta_{\mathbf{l}}$.

Повертаючись до (21), знаходимо

$\Delta_{g}^{(1)}=-\frac{3}{4} q t R_{2}^{3}\left(\frac{\varphi(x)}{a_{4}^{\prime}}\right)^{2}\left\{N^{\prime} F_{0}-6 R_{2} A_{0} \times\right.$

$\times \frac{1}{2} \sum_{k \leq B_{1}} \rho_{\mathbf{k}} \rho_{-\mathbf{k}}+3 R_{2} B_{0} \frac{1}{2} \sum_{k \leq B_{1}}\left(c^{\prime}\right)^{2} k^{2} \rho_{\mathbf{k}} \rho_{-\mathbf{k}}+3 s^{-3} \times$

$\left.\times R_{2}^{2} C_{0} \frac{1}{2}\left(N_{1}\right)^{-1} \sum_{k_{1}, \ldots, k_{4} \leq B_{1}} \rho_{\mathbf{k}_{1}} \cdots \rho_{\mathbf{k}_{4}} \delta_{\mathbf{k}_{1}+\cdots+\mathbf{k}_{4}}\right\}$.

Величини $F_{0}, A_{0}, B_{0}$ та $C_{0}$ задаються співвідношеннями

$$
\begin{aligned}
F_{0} & =-\sum_{\bar{x} \neq 0} Y^{3}(\bar{x})(Y(\bar{x})-\mathcal{Z}(\bar{x})) \rho(\bar{x}), \\
A_{0} & =\sum_{\bar{x} \neq 0} Y^{2}(\bar{x})(Y(\bar{x})-\mathcal{Z}(\bar{x})) \rho(\bar{x}), \\
B_{0} & =\sum_{\bar{x} \neq 0} Y^{2}(\bar{x})(Y(\bar{x})-\mathcal{Z}(\bar{x})) \bar{x}^{2} \rho_{1}(\bar{x}), \\
C_{0} & =-\sum_{\bar{x} \neq 0} Y(\bar{x})(Y(\bar{x})-\mathcal{Z}(\bar{x})) \rho(\bar{x}) .
\end{aligned}
$$

При підсумовуванні в (26) враховується $\bar{x}-$ відстань до частинок $(\bar{x}=1, \sqrt{2}, \sqrt{3}, \ldots)$. До складу $(26)$, крім $Y(\bar{x})$ і $\mathcal{Z}(\bar{x})$, входять кількість частинок $\rho(\bar{x})$, які розміщені у вузлах простої кубічної гратки на відстані $\bar{x}$ від початку координат, а також кількість тих самих частинок $\rho_{1}(\bar{x})=\sum_{\Theta} \rho_{\Theta}(\bar{x}) \cos ^{2} \Theta$ з врахуванням ї кутового розподілу. Через $\rho_{\Theta}(\bar{x})$ позначено кількість частинок, для яких $\cos ^{2} \Theta$ набуває однакових значень $(\Theta-$ кут між віссю $O z$ і напрямком на частинку). Відзначимо, що $\rho(\bar{x})=\sum_{\Theta} \rho_{\Theta}(\bar{x})$. Значення $\rho(\bar{x}), \rho_{\Theta}(\bar{x})$ та $\rho_{1}(\bar{x})$ залежно від $\bar{x}$ подано в $[11,22]$. Величини $F_{0}, A_{0}, B_{0}, C_{0}$ отримуються в результаті врахування поправки на усереднення і приводять до виникнення відмінного від нуля критичного показника кореляційної функції. Їхні числові оцінки наведено в [11] для проміжних значень параметра РГ $s$, близьких до значення $s=s^{*}=3,5862$, яке при $\Delta \tilde{\Phi}(k)=0$ відповідає рівному нулю у фіксованій точці середньому значенню коефіцієнта при другому степені змінної ефективної густини міри. Саме такі $s$ є оптимальними для даної методики розрахунку. Це зв'язано з двома обставинами. По-перше, при малих значеннях $s$ в системі необхідно враховувати наявність одиничного елемента. Для його виділення РС слід представити у вигляді рядів теорії збурень відносно гаусового розподілу $[21,24]$. По-друге, при великих значеннях $s$ виникають великі інтервали хвильових векторів, в яких проводиться усереднення $\tilde{\Phi}(k)$. При цьому поправка $\beta \tilde{\Phi}(k)-\beta \tilde{\Phi}\left(B_{n+1}, B_{n}\right)$ є суттєвою і ї̈ врахування в лінійному наближенні некоректне.

Слід підкреслити, що врахування наступних доданків в (16), пропорційних до вищих порядків операторів $\partial / \partial \rho_{\mathbf{k}}$, не приводить до зміни функціонального вигляду виразу для $\Delta_{g}^{(1)}(25)$ (див. $\left.[11,22]\right)$. Іншими стають коефіцієнти $F_{0}, A_{0}, B_{0}$ і $C_{0}$. В подальших розрахунках будемо нехтувати подібним перенормуванням цих коефіцієнтів.

Після послідовного інтегрування в нульовому, першому, ..., n-му шарах фазового простору КЗ приходимо до такого виразу для статистичної суми системи в лінійному наближенні за $\Delta \tilde{\Phi}(k)$ :

$$
\begin{aligned}
& Z=2^{N} 2^{\left(N_{n+1}-1\right) / 2} \tilde{Q}_{0} \tilde{Q}_{1} \cdots \tilde{Q}_{n}\left[Q\left(P_{n}\right)\right]^{N_{n+1}} \times \\
& \times \int \exp \left\{-\frac{1}{2} \sum_{k \leq B_{n+1}} \tilde{d}_{n+1}(k) \rho_{\mathbf{k}} \rho_{-\mathbf{k}}-\frac{1}{4 !}\left(N_{n+1}\right)^{-1} \times\right. \\
& \left.\times \sum_{k_{1}, \ldots, k_{4} \leq B_{n+1}} \tilde{a}_{4}^{(n+1)} \rho_{\mathbf{k}_{1}} \cdots \rho_{\mathbf{k}_{4}} \delta_{\mathbf{k}_{1}+\cdots+\mathbf{k}_{4}}\right\}(d \rho)^{N_{n+1}} .
\end{aligned}
$$

Порівняно з результатами (див., наприклад, [8,9,11]), одержаними раніше без врахування поправки на усереднення потенціалу, в (27) виникають нові величини. Зокрема,

$f\left(x_{n}\right)=-\frac{3 \sqrt{3}}{4} s^{6} U\left(x_{n}\right) \frac{U^{3}\left(y_{n}\right)}{y_{n}} \frac{q_{n} t_{n}}{\sqrt{\tilde{a}_{4}^{(n)}}} F_{0}$ 
характеризує поправку до парціальних статистичних сум, які тепер набувають вигляду

$$
\begin{aligned}
& \tilde{Q}_{0}=\left[Q_{0} e^{f(x)}\right]^{N^{\prime}}, \quad \tilde{Q}_{1}=\left[(3 / \varphi(y))^{1 / 4} e^{\left(x_{1}^{2}+y^{2}\right) / 4} \times\right. \\
& \left.\times U\left(0, x_{1}\right) U(0, y) e^{f\left(x_{1}\right)}\right]^{N_{1}}, \ldots, \\
& \tilde{Q}_{n}=\left[\left(3 / \varphi\left(y_{n-1}\right)\right)^{1 / 4} e^{\left(x_{n}^{2}+y_{n-1}^{2}\right) / 4} U\left(0, x_{n}\right) \times\right. \\
& \left.\times U\left(0, y_{n-1}\right) e^{f\left(x_{n}\right)}\right]^{N_{n}} .
\end{aligned}
$$

Аргументами функцій тут виступають

$x_{n}=\sqrt{3} \tilde{d}_{n}\left(B_{n+1}, B_{n}\right)\left(\tilde{a}_{4}^{(n)}\right)^{-1 / 2}$,

$y_{n-1}=s^{3 / 2} U\left(x_{n-1}\right)\left(3 / \varphi\left(x_{n-1}\right)\right)^{1 / 2}$.

Вираз для $f\left(x_{n}\right)(28)$, крім $q_{n}=q \frac{1+\alpha_{0}}{s^{2}} \frac{1+\alpha_{1}}{s^{2}} \cdots$ $\cdots \frac{1+\alpha_{n-1}}{s^{2}}$, містить величину

$t_{n}=\sqrt{\frac{\tilde{a}_{4}^{(n)}}{3}} \frac{1}{U\left(x_{n}\right)} \frac{s^{2}}{1+\alpha_{0}} \frac{s^{2}}{1+\alpha_{1}} \cdots \frac{s^{2}}{1+\alpha_{n-1}} t_{0}^{(n)} \times$

$$
\times \frac{1}{\beta \tilde{\Phi}(0)} \text {, }
$$

де

$t_{0}^{(n)}=\frac{3}{1+s^{-1}+s^{-2}}+\frac{3 a_{n}}{1-s^{-3}} I_{0}$.

Для $I_{0}$ справедлива відповідна формула із $(24)$, в яку замість $а$ слід підставити

$a_{n}=\bar{q}-\sqrt{\frac{\tilde{a}_{4}^{(n)}}{3}} \frac{1}{U\left(x_{n}\right)} \frac{s^{2}}{1+\alpha_{0}} \frac{s^{2}}{1+\alpha_{1}} \cdots \frac{s^{2}}{1+\alpha_{n-1}} \times$

$$
\times \frac{1}{\beta \tilde{\Phi}(0)} \text {. }
$$

Величина

$\alpha_{n}=\frac{9 \pi^{2}}{4} s^{6} U^{4}\left(y_{n}\right) \bar{q} t_{n} B_{0}$

визначає поправку на усереднення потенціалу в $n$-му шарі фазового простору КЗ. Зазначимо, що в даній роботі використовуються позначення $x_{0} \equiv x, y_{0} \equiv y$, $q_{0} \equiv q, t_{0} \equiv t, a_{0} \equiv a$.

Коефіцієнти $\tilde{d}_{n+1}$ та $\tilde{a}_{4}^{(n+1)}$ задовольняють такі РС: $\tilde{d}_{n+1}\left(B_{n+2}, B_{n+1}\right)=\tilde{d}_{n}\left(B_{n+1}, B_{n}\right) \tilde{N}\left(x_{n}\right)-$ $-q \frac{1+\alpha_{0}}{s^{2}} \frac{1+\alpha_{1}}{s^{2}} \cdots \frac{1+\alpha_{n-1}}{s^{2}}\left(1-\frac{1+\alpha_{n}}{s^{2}}\right)$, $\tilde{a}_{4}^{(n+1)}=\tilde{a}_{4}^{(n)} s^{-3} \tilde{E}\left(x_{n}\right)$.
Поправки від врахування внеску на усереднення потенціалу у виразах

$\tilde{N}\left(x_{n}\right)=N\left(x_{n}\right)\left(1-G\left(x_{n}\right) A_{0}\right)$,

$\tilde{E}\left(x_{n}\right)=E\left(x_{n}\right)\left(1+\mathcal{K}\left(x_{n}\right) C_{0}\right)$

задано доданками $G\left(x_{n}\right) A_{0}$ та $\mathcal{K}\left(x_{n}\right) C_{0}$. Тут

$G\left(x_{n}\right)=\frac{9 \sqrt{3}}{2} s^{6} \frac{U^{3}\left(y_{n}\right)}{y_{n}} U\left(x_{n}\right) \frac{q t_{n}}{\sqrt{\tilde{u}_{n}}}$,

$\mathcal{K}\left(x_{n}\right)=27 \sqrt{3} s^{3} \frac{U^{5}\left(y_{n}\right)}{y_{n} \varphi\left(y_{n}\right)} U\left(x_{n}\right) \frac{q t_{n}}{\sqrt{\tilde{u}_{n}}}$.

В термінах

$\tilde{r}_{n}=\frac{s^{2}}{1+\alpha_{0}} \frac{s^{2}}{1+\alpha_{1}} \cdots \frac{s^{2}}{1+\alpha_{n-1}} \tilde{d}_{n}(0)$,

$\tilde{u}_{n}=\frac{s^{4}}{\left(1+\alpha_{0}\right)^{2}} \frac{s^{4}}{\left(1+\alpha_{1}\right)^{2}} \cdots \frac{s^{4}}{\left(1+\alpha_{n-1}\right)^{2}} \tilde{a}_{4}^{(n)}$

РC (34) набувають вигляду

$\tilde{r}_{n+1}=\frac{s^{2}}{1+\alpha_{n}}\left[\left(\tilde{r}_{n}+q\right) \tilde{N}\left(x_{n}\right)-q\right]$,

$\tilde{u}_{n+1}=\frac{s}{\left(1+\alpha_{n}\right)^{2}} \tilde{u}_{n} \tilde{E}\left(x_{n}\right)$,

а для $t_{n}(31)$ маємо

$t_{n}=\sqrt{\frac{\tilde{u}_{n}}{3}} \frac{1}{U\left(x_{n}\right)} t_{0}^{(n)} \frac{1}{\beta \tilde{\Phi}(0)}$,

$t_{0}^{(n)}=\frac{3}{1+s^{-1}+s^{-2}}+\frac{3 a_{n}}{1-s^{-3}} \int_{1 / s}^{1} \frac{d k}{k^{2}-a_{n}}$,

де

$a_{n}=\bar{q}-\sqrt{\frac{\tilde{u}_{n}}{3}} \frac{1}{U\left(x_{n}\right)} \frac{1}{\beta \tilde{\Phi}(0)}$.

Одержані РС (38) містять дві суттєві відмінності від РС [11, 25], які мають місце без врахування поправки на $\Delta \Phi(k)$. Перша з них полягає в специфічній заміні змінних (37), що включає в себе множники $\left(1+\alpha_{0}\right)\left(1+\alpha_{1}\right) \cdots\left(1+\alpha_{n-1}\right)$ і цим відрізняється від відповідної заміни без врахування поправки. Друга відмінність стосується переходу спеціальних функцій $N\left(x_{n}\right)$ та $E\left(x_{n}\right)(10)$ в $\tilde{N}\left(x_{n}\right)$ та $\tilde{E}\left(x_{n}\right)(35)$. Ця відмінність, як буде видно далі, приводить до зсуву координат фіксованої точки і пов'язана з поправками до критичних показників термодинамічних функцій. 


\section{4. Дослідження РС}

Як частковий розв'язок $\mathrm{PC}(38)$ мають нову фіксовану точку $(\tilde{r}, \tilde{u})$, яка при $\Delta \tilde{\Phi}(k) \neq 0$ відрізняється від фіксованої точки $\left(r^{(0)}, u^{(0)}\right)[11,25,26]$ у випадку $\Delta \tilde{\Phi}(k)=0$. Справді, припускаючи, що

$\tilde{r}_{n+1}=\tilde{r}_{n}=\tilde{r}, \quad \tilde{u}_{n+1}=\tilde{u}_{n}=\tilde{u}$,

із другого рівняння (38) одержуємо рівність

$\tilde{E}(\tilde{x})=\frac{\left(1+\alpha^{(0)}\right)^{2}}{s}$,

3 якої знаходимо величину

$\tilde{x}=\sqrt{3}(\tilde{r}+q)(\tilde{u})^{-1 / 2}$.

Точність розрахунку величини $\tilde{x}$ має відповідати наближенню, в якому отримані РС (38). Тому в лінійному наближенні за $\Delta \tilde{\Phi}(k)$ приходимо до формул

$\tilde{x}=x^{(0)}-\frac{\mathcal{K}\left(x^{(0)}\right) C_{0}-2 \alpha^{(0)}}{\psi\left(x^{(0)}\right)}, \psi\left(x^{(0)}\right)=\frac{E^{\prime}\left(x^{(0)}\right)}{E\left(x^{(0)}\right)}$.

Тут величина $x^{(0)}$ є розв'язком рівняння $E\left(x^{(0)}\right)=$ $s^{-1}$ і характеризує аргумент $x_{n}$ в фіксованій точці, отриманій без врахування залежності фур'є-образу потенціалу від хвильового вектора. Для $\alpha^{(0)}$ та $\mathcal{K}\left(x^{(0)}\right)$ маємо

$\alpha^{(0)}=\frac{9 \pi^{2}}{4} s^{6} U^{4}\left(y^{(0)}\right) \bar{q} t^{(0)} B_{0}$,

$\mathcal{K}\left(x^{(0)}\right)=27 \sqrt{3} s^{3} \frac{U^{5}\left(y^{(0)}\right)}{y^{(0)} \varphi\left(y^{(0)}\right)} U\left(x^{(0)}\right) \frac{q t^{(0)}}{\sqrt{u^{(0)}}}$,

де $t^{(0)}=t_{n}\left(u^{(0)}, x^{(0)}\right)$, а $y^{(0)}$ визначається виразом

$y^{(0)}=s^{3 / 2} U\left(x^{(0)}\right)\left(3 / \varphi\left(x^{(0)}\right)\right)^{1 / 2}$.

Координати фіксованої точки представимо у вигляді $\tilde{r}=-\tilde{f} \beta \tilde{\Phi}(0), \quad \tilde{u}=\tilde{\varphi}(\beta \Phi(0))^{2}$,

Т а б л и ц я 1. Величина $\alpha^{(0)}$ із (45), зміщення основного аргумента $\boldsymbol{x}_{n}$ в фіксованій точці $\Delta x=\tilde{x}-x^{(0)}$ та величини $\tilde{f}$ i $\tilde{\varphi}$, які характеризують координати фіксованої точки (46) при врахуванні поправки на усереднення потенціалу, а також власні значення $\tilde{E}_{1}, \tilde{E}_{2}$ матриці перетворення $\tilde{\mathcal{R}}$ із (51)

\begin{tabular}{c|c|c|c|c|c|c}
\hline$s$ & $\alpha^{(0)}$ & $\Delta x$ & $\tilde{f}$ & $\tilde{\varphi}$ & $\tilde{E}_{1}$ & $\tilde{E}_{2}$ \\
\hline 3 & 0,0113 & 0,2551 & 0,3635 & 0,4975 & 6,8596 & 0,4109 \\
$s^{*}$ & 0,0235 & 0,3332 & 0,4308 & 0,6353 & 9,2168 & 0,3801 \\
4 & 0,0330 & 0,3782 & 0,4709 & 0,7261 & 11,0407 & 0,3649 \\
5 & 0,0595 & 0,5047 & 0,5143 & 0,8630 & 16,1822 & 0,3381 \\
\hline
\end{tabular}

де

$$
\begin{aligned}
& \tilde{f}=\bar{q} \frac{\tilde{N}(\tilde{x})-1}{\tilde{N}(\tilde{x})-\left(1+\alpha^{(0)}\right) / s^{2}} \\
& \tilde{\varphi}=3 \bar{q}^{2}\left[\frac{s^{2}-\alpha^{(0)}-1}{s^{2} \tilde{n}(\tilde{x})-\tilde{x}\left(1+\alpha^{(0)}\right)}\right]^{2} .
\end{aligned}
$$

Тут введено позначення

$\tilde{n}(\tilde{x})=n(\tilde{x})\left(1-G(\tilde{x}) A_{0}\right)$,

$n(\tilde{x})=\frac{\tilde{y} U(\tilde{y})}{U(\tilde{x})}$,

$G(\tilde{x})=\frac{9 \sqrt{3}}{2} s^{6} \frac{U^{3}(\tilde{y})}{\tilde{y}} U(\tilde{x}) \frac{\bar{q} \tilde{t}}{\sqrt{\tilde{\varphi}}}$,

а також $\tilde{t}=t_{n}(\tilde{\varphi}, \tilde{x})$. Для $\tilde{f}$ і $\tilde{\varphi}$ в лінійному наближенні за $\Delta \tilde{\Phi}(k)$ отримуємо

$$
\begin{aligned}
& \tilde{f}=f_{0}\left\{1+\frac{\alpha^{(0)} x^{(0)} / s^{2}}{n\left(x^{(0)}\right)-x^{(0)} / s^{2}}+\right. \\
& +\frac{\left[x^{(0)} n^{\prime}\left(x^{(0)}\right)-n\left(x^{(0)}\right)\right] \Delta x-n\left(x^{(0)}\right) G\left(x^{(0)}\right) A_{0} x^{(0)}}{\left[n\left(x^{(0)}\right)-x^{(0)}\right]\left[n\left(x^{(0)}\right)-x^{(0)} / s^{2}\right]} \times \\
& \left.\times\left(1-s^{-2}\right)\right\}, \\
& \tilde{\varphi}=\varphi_{0}\left\{1-\frac{2 \alpha^{(0)} / s^{2}}{1-s^{-2}}+\right. \\
& \left.+2 \frac{\left[-n^{\prime}\left(x^{(0)}\right)+s^{-2}\right] \Delta x+n\left(x^{(0)}\right) G\left(x^{(0)}\right) A_{0}+x^{(0)} \alpha^{(0)} / s^{2}}{n\left(x^{(0)}\right)-x^{(0)} / s^{2}}\right\} .
\end{aligned}
$$

Величини $f_{0}, \varphi_{0}$ характеризують координати фіксованої точки без врахування залежності $\tilde{\Phi}(k)$ від хвильового вектора [11, 19], $\Delta x=\tilde{x}-x^{(0)}$, а для $n^{\prime}\left(x^{(0)}\right)$ та $G\left(x^{(0)}\right)$ відповідно маємо

$$
\begin{aligned}
& n^{\prime}\left(x^{(0)}\right)=\left.n(x)\left[\frac{y^{\prime}}{y}+y^{\prime} \frac{U^{\prime}(y)}{U(y)}-\frac{U^{\prime}(x)}{U(x)}\right]\right|_{\substack{x=x^{(0)} \\
y=y^{(0)}}}, \\
& G\left(x^{(0)}\right)=\frac{9 \sqrt{3}}{2} s^{6} \frac{U^{3}\left(y^{(0)}\right)}{y^{(0)}} U\left(x^{(0)}\right) \frac{q t^{(0)}}{\sqrt{u^{(0)}}}
\end{aligned}
$$

У табл. 1 подано $\alpha^{(0)}, \Delta x, \tilde{f}, \tilde{\varphi}$ для деяких проміжних значень параметра РГ $s$.

Здійснимо лінеаризацію РС (38) в околі фіксованої точки

$$
\left(\begin{array}{c}
\tilde{r}_{n+1}-\tilde{r} \\
\tilde{u}_{n+1}-\tilde{u}
\end{array}\right)=\tilde{\mathcal{R}}\left(\begin{array}{c}
\tilde{r}_{n}-\tilde{r} \\
\tilde{u}_{n}-\tilde{u}
\end{array}\right)
$$

та знайдемо елементи матриці цього перетворення. Загальні вирази для елементів $\tilde{R}_{i j}$ матриці $\tilde{\mathcal{R}}$ набува- 
ють вигляду

$\tilde{R}_{11}=\frac{s^{2}}{1+\tilde{\alpha}} n(\tilde{x})\left[T(\tilde{x})-G(\tilde{x}) A_{0}(\Pi(\tilde{x})+T(\tilde{x}))\right]$,

$\tilde{R}_{12}=\frac{s^{2}}{1+\tilde{\alpha}} \frac{n(\tilde{x})(\tilde{u})^{-1 / 2}}{2 \sqrt{3}}\left[1-\tilde{x} T(\tilde{x})+G(\tilde{x}) \tilde{x} A_{0} \times\right.$

$\times(\Pi(\tilde{x})+T(\tilde{x}))]$,

$\tilde{R}_{21}=\frac{s E(\tilde{x})}{(1+\tilde{\alpha})^{2}}(3 \tilde{u})^{1 / 2}\left[\psi(\tilde{x})+\mathcal{K}(\tilde{x}) C_{0} \times\right.$

$\left.\times\left(\psi(\tilde{x})+\Pi_{1}(\tilde{x})\right)\right]$,

$\tilde{R}_{22}=\frac{s E(\tilde{x})}{(1+\tilde{\alpha})^{2}}\left\{1-\frac{1}{2} \tilde{x} \psi(\tilde{x})+\frac{1}{2} \mathcal{K}(\tilde{x}) C_{0} \times\right.$

$\left.\times\left[1-\tilde{x}\left(\psi(\tilde{x})+\Pi_{1}(\tilde{x})\right)\right]\right\}$,

де $\tilde{\alpha}=\alpha_{n}(\tilde{\varphi}, \tilde{x})$ і використано такі позначення:

$T(\tilde{x})=\frac{\tilde{y}^{\prime}}{\tilde{y}}+\tilde{y}^{\prime} \frac{U^{\prime}(\tilde{y})}{U(\tilde{y})}-\frac{U^{\prime}(\tilde{x})}{U(\tilde{x})}=\left.\frac{1}{n(\tilde{x})}\left[\frac{\partial n\left(x_{n}\right)}{\partial x_{n}}\right]\right|_{\substack{x_{n}=\tilde{x} \\ y_{n}=\tilde{y}}}$,

$\Pi(\tilde{x})=-\frac{\tilde{y}^{\prime}}{\tilde{y}}+3 \tilde{y}^{\prime} \frac{U^{\prime}(\tilde{y})}{U(\tilde{y})}+\frac{U^{\prime}(\tilde{x})}{U(\tilde{x})}=\left.\frac{1}{G(\tilde{x})}\left[\frac{\partial G\left(x_{n}\right)}{\partial x_{n}}\right]\right|_{\substack{x_{n}=\tilde{x} \\ y_{n}=\tilde{y}}}$,

$\psi(\tilde{x})=\tilde{y}^{\prime} \frac{\varphi^{\prime}(\tilde{y})}{\varphi(\tilde{y})}-\frac{\varphi^{\prime}(\tilde{x})}{\varphi(\tilde{x})}=\left.\frac{1}{E(\tilde{x})}\left[\frac{\partial E\left(x_{n}\right)}{\partial x_{n}}\right]\right|_{\substack{x_{n}=\tilde{x} \\ y_{n}=\tilde{y}}}$,

$\Pi_{1}(\tilde{x})=-\frac{\tilde{y}^{\prime}}{\tilde{y}}+\tilde{y}^{\prime}\left[5 \frac{U^{\prime}(\tilde{y})}{U(\tilde{y})}-\frac{\varphi^{\prime}(\tilde{y})}{\varphi(\tilde{y})}\right]+\frac{U^{\prime}(\tilde{x})}{U(\tilde{x})}=$

$=\left.\frac{1}{\mathcal{K}(\tilde{x})}\left[\frac{\partial \mathcal{K}\left(x_{n}\right)}{\partial x_{n}}\right]\right|_{\substack{x_{n}=\tilde{x} \\ y_{n}=\tilde{y}}}$

$\mathcal{K}(\tilde{x})=27 \sqrt{3} s^{3} \frac{U^{5}(\tilde{y})}{\tilde{y} \varphi(\tilde{y})} U(\tilde{x}) \frac{\bar{q} \tilde{t}}{\sqrt{\tilde{\varphi}}}$.

Як і раніше, всі обчислення виконуватимемо в наближенні лінійного внеску від поправки $\Delta \tilde{\Phi}(k)$. В цьому наближенні для матричного елемента $\tilde{R}_{11}$ із (52) одержуємо

$\tilde{R}_{11}=R_{11}\left(1-\alpha^{(0)}\right)+R_{11}^{(1)} \Delta x+R_{11}^{(2)} A_{0}$,

де

$R_{11}=s^{2} n\left(x^{(0)}\right) T\left(x^{(0)}\right)$,

$R_{11}^{(1)}=s^{2}\left[n\left(x^{(0)}\right) T^{\prime}\left(x^{(0)}\right)+n^{\prime}\left(x^{(0)}\right) T\left(x^{(0)}\right)\right]$,

$R_{11}^{(2)}=-s^{2} n\left(x^{(0)}\right) G\left(x^{(0)}\right)\left[\Pi\left(x^{(0)}\right)+T\left(x^{(0)}\right)\right]$.

Тут похідна $T^{\prime}\left(x^{(0)}\right)$ обчислюється за формулою

$T^{\prime}\left(x^{(0)}\right)=\left\{y^{\prime \prime}\left(\frac{1}{y}+\frac{U^{\prime}(y)}{U(y)}\right)+\left(y^{\prime}\right)^{2}\left[\frac{U^{\prime \prime}(y)}{U(y)}-\right.\right.$

$\left.\left.-\left(\frac{U^{\prime}(y)}{U(y)}\right)^{2}-\frac{1}{y^{2}}\right]-\frac{U^{\prime \prime}(x)}{U(x)}+\left(\frac{U^{\prime}(x)}{U(x)}\right)^{2}\right\}\left.\right|_{\substack{x=x^{(0)} \\ y=y^{(0)}}}$, в якій

$$
\begin{aligned}
& y^{\prime \prime}=y^{\prime}\left[\frac{U^{\prime}(x)}{U(x)}-\frac{1}{2} \frac{\varphi^{\prime}(x)}{\varphi(x)}\right]+y\left[\frac{U^{\prime \prime}(x)}{U(x)}-\left(\frac{U^{\prime}(x)}{U(x)}\right)^{2}-\right. \\
& \left.-\frac{1}{2} \frac{\varphi^{\prime \prime}(x)}{\varphi(x)}+\frac{1}{2}\left(\frac{\varphi^{\prime}(x)}{\varphi(x)}\right)^{2}\right] .
\end{aligned}
$$

Запишемо матричні елементи $\tilde{R}_{12}, \tilde{R}_{21}$ у вигляді

$\tilde{R}_{12}=\tilde{R}_{12}^{(0)}(\tilde{u})^{-1 / 2}, \quad \tilde{R}_{21}=\tilde{R}_{21}^{(0)}(\tilde{u})^{1 / 2}$.

Тоді для $\tilde{R}_{12}^{(0)}$ знаходимо

$\tilde{R}_{12}^{(0)}=R_{12}^{(0)}\left(1-\alpha^{(0)}\right)+R_{12}^{(1)} \Delta x+R_{12}^{(2)} A_{0}$,

де

$R_{12}^{(0)}=\frac{s^{2}}{2 \sqrt{3}} n\left(x^{(0)}\right)\left[1-x^{(0)} T\left(x^{(0)}\right)\right]$,

$R_{12}^{(1)}=\frac{s^{2}}{2 \sqrt{3}}\left\{n^{\prime}\left(x^{(0)}\right)\left[1-x^{(0)} T\left(x^{(0)}\right)\right]-n\left(x^{(0)}\right) \times\right.$

$\left.\times\left[T\left(x^{(0)}\right)+x^{(0)} T^{\prime}\left(x^{(0)}\right)\right]\right\}$,

$R_{12}^{(2)}=\frac{s^{2}}{2 \sqrt{3}} n\left(x^{(0)}\right) G\left(x^{(0)}\right) x^{(0)}\left[\Pi\left(x^{(0)}\right)+T\left(x^{(0)}\right)\right]$,

а для $\tilde{R}_{21}^{(0)}$ будемо мати

$\tilde{R}_{21}^{(0)}=R_{21}^{(0)}\left(1-2 \alpha^{(0)}\right)+R_{21}^{(1)} \Delta x+R_{21}^{(2)} C_{0}$,

де

$R_{21}^{(0)}=s \sqrt{3} E\left(x^{(0)}\right) \psi\left(x^{(0)}\right)$,

$R_{21}^{(1)}=s \sqrt{3} E\left(x^{(0)}\right)\left[\psi^{\prime}\left(x^{(0)}\right)+\psi^{2}\left(x^{(0)}\right)\right]$,

$R_{21}^{(2)}=s \sqrt{3} E\left(x^{(0)}\right) \mathcal{K}\left(x^{(0)}\right)\left[\psi\left(x^{(0)}\right)+\Pi_{1}\left(x^{(0)}\right)\right]$.

Тут

$\psi^{\prime}\left(x^{(0)}\right)=\left\{y^{\prime \prime} \frac{\varphi^{\prime}(y)}{\varphi(y)}+\left(y^{\prime}\right)^{2}\left[\frac{\varphi^{\prime \prime}(y)}{\varphi(y)}-\left(\frac{\varphi^{\prime}(y)}{\varphi(y)}\right)^{2}\right]+\right.$

$\left.+\left(\frac{\varphi^{\prime}(x)}{\varphi(x)}\right)^{2}-\frac{\varphi^{\prime \prime}(x)}{\varphi(x)}\right\}\left.\right|_{\substack{x=x^{(0)} \\ y=y^{(0)}}}$.

Матричний елемент $\tilde{R}_{22}$ задовольняє співвідношення

$\tilde{R}_{22}=R_{22}\left(1-2 \alpha^{(0)}\right)+R_{22}^{(1)} \Delta x+R_{22}^{(2)} C_{0}$, 
де

$R_{22}=s E\left(x^{(0)}\right)\left[1-\frac{1}{2} x^{(0)} \psi\left(x^{(0)}\right)\right]$

$R_{22}^{(1)}=s E\left(x^{(0)}\right)\left\{\psi\left(x^{(0)}\right)\left[1-\frac{1}{2} x^{(0)} \Psi\left(x^{(0)}\right)\right]-\right.$

$\left.-\frac{1}{2}\left[\psi\left(x^{(0)}\right)+x^{(0)} \psi^{\prime}\left(x^{(0)}\right)\right]\right\}$,

$R_{22}^{(2)}=\frac{s E\left(x^{(0)}\right)}{2} \mathcal{K}\left(x^{(0)}\right)\left\{1-x^{(0)}\left[\psi\left(x^{(0)}\right)+\Pi_{1}\left(x^{(0)}\right)\right]\right\}$.

Зауважимо, що величини $R_{11}, R_{12}^{(0)}, R_{21}^{(0)}, R_{22}$ в (54)(65) збігаються з виразами для матричних елементів, отриманих у роботах $[11,19]$ без врахування поправки на усереднення потенціалу. Пропорційні до $R_{i j}^{(1)} \Delta x$ внески в матричні елементи $\tilde{R}_{i j}$ відповідають зсуву фіксованої точки за рахунок врахування залежності фур'є-образу потенціалу взаємодії від хвильового вектора. Доданки типу $\tilde{R}_{i j}^{(2)} A_{0}$ та $\tilde{R}_{i j}^{(2)} C_{0}$ описують прямий внесок у $\tilde{R}_{i j}$ від поправки на усереднення. Фіксована точка продовжує залишатись сідловою, оскільки для власних значень матриці $\tilde{\mathcal{R}}$ виконуються нерівності $\tilde{E}_{1}>1, \tilde{E}_{2}<1$ (див. табл. 1$)$.

Розрахувавши власні вектори і власні значення матриці перетворення $\tilde{\mathcal{R}}$ із (51), знаходимо явні розв'язки $\mathrm{PC} \mathrm{(38):}$

$\tilde{r}_{n}=\tilde{r}+\tilde{c}_{1} \tilde{E}_{1}^{n}+\tilde{c}_{2} \tilde{R} \tilde{E}_{2}^{n}$,

$\tilde{u}_{n}=\tilde{u}+\tilde{c}_{1} \tilde{R}_{1} \tilde{E}_{1}^{n}+\tilde{c}_{2} \tilde{E}_{2}^{n}$.

Тут

$\tilde{c}_{1}=\left[r^{\prime}-\tilde{r}-\left(a_{4}^{\prime}-\tilde{u}\right) \tilde{R}\right] / \tilde{D}, \quad \tilde{R}=\tilde{R}_{12} /\left(\tilde{E}_{2}-\tilde{R}_{11}\right)$,

$\tilde{c}_{2}=\left[a_{4}^{\prime}-\tilde{u}-\left(r^{\prime}-\tilde{r}\right) \tilde{R}_{1}\right] / \tilde{D}, \tilde{R}_{1}=\left(\tilde{E}_{1}-\tilde{R}_{11}\right) / \tilde{R}_{12}$,

$\tilde{D}=\left(\tilde{E}_{1}-\tilde{E}_{2}\right) /\left(\tilde{R}_{11}-\tilde{E}_{2}\right), \quad r^{\prime}=a_{2}^{\prime}-\beta \tilde{\Phi}(0)$,

вихідні коефіцієнти $a_{2}^{\prime}$ та $a_{4}^{\prime}$ задані в (6), а $\tilde{r}$ і $\tilde{u}$ визначені в $(46),(49)$. Враховуючи позначення (37), приходимо до співвідношень

$\tilde{d}_{n}\left(B_{n+1}, B_{n}\right)=s^{-2 n}\left[\prod_{m=0}^{n-1}\left(1+\alpha_{m}\right)\right] \times$

$\times\left[\tilde{r}+q+\tilde{c}_{1} \tilde{E}_{1}^{n}+\tilde{c}_{2} \tilde{R} \tilde{E}_{2}^{n}\right]$,

$\tilde{a}_{4}^{(n)}=s^{-4 n}\left[\prod_{m=0}^{n-1}\left(1+\alpha_{m}\right)^{2}\right]\left[\tilde{u}+\tilde{c}_{1} \tilde{R}_{1} \tilde{E}_{1}^{n}+\tilde{c}_{2} \tilde{E}_{2}^{n}\right]$.

Основною відмінністю розв'язків (68) від наведених раніше в роботах $[8,9,19]$ за відсутності поправки на усереднення потенціалу є наявність множників типу $\left(1+\alpha_{m}\right)$. В загальному випадку вони залежать від температури та номера блочної структури $m$. Однак у випадку $T=T_{c}$ поведінка коефіцієнтів $\tilde{d}_{n}$ та $\tilde{a}_{4}^{(n)}$ із (68) є особливою. Беручи до уваги те, що при $T=T_{c}$

$\lim _{m \rightarrow \infty} \alpha_{m}\left(T_{c}\right)=\alpha^{(0)}$,

одержуємо таку асимптотику цих величин за $n$ :

$\tilde{d}_{n}\left(B_{n+1}, B_{n}\right)=(\tilde{r}+q) s^{-n(2-\eta)}$,

$\tilde{a}_{4}^{(n)}=\tilde{u} s^{-2 n(2-\eta)}$.

Показник $\eta$ задається формулою

$\eta=\frac{\alpha^{(0)}}{\ln s}$

і відповідає критичному показнику кореляційної функції.

Отже, врахування поправки, пов'язаної з усередненням фур'є-образу потенціалу $\tilde{\Phi}(k)$ при інтегруванні статистичної суми системи, для окремих блочних структур приводить до зміни асимптотики коефіцієнтів $\tilde{d}_{n}$ та $\tilde{a}_{4}^{(n)}$ при $T=T_{c}$ (показники степенів в (69) поряд з $n$, на відміну від випадку $\Delta \tilde{\Phi}(k)=0$, містять $\eta)$.

\section{5. Вільна енергія системи}

Загальна схема розрахунку вільної енергії, розвинута в $[8,11,19,25]$, застосовна до випадку врахування поправки на усереднення потенціалу. Вільну енергію системи обчислюватимемо, розділяючи внески від коротко- і довгохвильових мод коливань спінової густини. Незважаючи на зміни в розв'язках РC (68), легко показати, що й надалі

$\tilde{c}_{1}=\tilde{c}_{10} \tau$,

де $\tau=\left(T-T_{c}\right) / T_{c}$, а $\tilde{c}_{10}$ - стала відмінна від нуля величина при $T=T_{c}$. Це дає змогу визначити $m_{\tau}$ - точку виходу системи із критичного режиму (KP). Аналогічно до результатів $[11,19,25]$ використаємо умову

$\tilde{d}_{m_{\tau+1}}(0)=0$

для знаходження точки виходу із КР флуктуацій при $T>T_{c}$. Приходимо до формули

$m_{\tau}=-\frac{\ln \tau}{\ln \tilde{E}_{1}}+m_{0}-1$.

Тут $\tilde{E}_{1}$ - більше власне значення матриці перетворення (51),

$m_{0}=\frac{\ln \left|\tilde{r} / \tilde{c}_{10}\right|}{\ln \tilde{E}_{1}}$. 
Розрахунок вільної енергї системи проілюструємо випадком температур, вищих від $T_{c}$. Вираз для вільної енергї при $T>T_{c}$ запишемо у вигляді

$F=F_{0}+F_{\mathrm{CR}}+F_{\mathrm{LGR}}$,

де $F_{0}=-k T N \ln 2$ відповідає вільній енергії невзаємодіючих спінів, $F_{\mathrm{CR}}$ - внеску у вільну енергію від короткохвильових мод коливань густини спінового моменту (область KP), а $F_{\mathrm{LGR}}$ - внеску від довгохвильових мод коливань (область граничного гаусового режиму $(\Gamma Г P))$. Для $F_{\mathrm{CR}}$ маємо

$F_{\mathrm{CR}}=-k T \sum_{n=0}^{m_{\tau}} \ln \tilde{Q}_{n}$.

Величину $\ln \tilde{Q}_{n}$ можна подати як

$\ln \tilde{Q}_{n}=\ln Q_{n}+N_{n} f\left(x_{n}\right)$.

Тут $Q_{n}$ - парціальна статистична сума $n$-ї блочної структури без врахування залежності фур'є-образу потенціалу $\tilde{\Phi}(k)$ від хвильового вектора, а $f\left(x_{n}\right)$ описує додатковий внесок, який виникає при врахуванні $\Delta \tilde{\Phi}(k)$. Його зручно записати у вигляді

$f\left(x_{n}\right)=-\frac{3 \sqrt{3}}{4} s^{6} F_{0} q \alpha_{n}^{\prime} t_{n}$,

де для величини $\alpha_{n}^{\prime}$ маємо вираз

$\alpha_{n}^{\prime}=\frac{1}{\sqrt{\tilde{u}_{n}}} U\left(x_{n}\right) U^{3}\left(y_{n-1}\right) y_{n-1}^{-1}$.

В околі фіксованої точки величина $\alpha_{n}^{\prime}$ задовольняє співвідношення

$$
\begin{aligned}
& \alpha_{n}^{\prime}=\frac{1}{\sqrt{\tilde{u}_{n}}} \frac{U\left(x^{(0)}\right)}{\left(y^{(0)}\right)^{4}}\left(1-\frac{9}{2} \frac{1}{\left(y^{(0)}\right)^{2}}\right) \times \\
& \times\left[1+A_{1}^{\prime \prime}\left(x_{n-1}-x^{(0)}\right)+A_{2}^{\prime \prime}\left(x_{n-1}-x^{(0)}\right)^{2}+\right. \\
& +A_{3}^{\prime \prime}\left(x_{n}-x^{(0)}\right)+A_{4}^{\prime \prime}\left(x_{n}-x^{(0)}\right)^{2}+ \\
& \left.+A_{5}^{\prime \prime}\left(x_{n-1}-x^{(0)}\right)\left(x_{n}-x^{(0)}\right)\right] .
\end{aligned}
$$

Тут використано позначення

$$
\begin{aligned}
& A_{l}^{\prime \prime}=\omega_{0} A_{l}^{\prime}, \\
& \omega_{0}=-\frac{3 \sqrt{3}}{4} s^{6} F_{0} t^{(0)} \bar{q} \frac{1}{\sqrt{\varphi_{0}}} \frac{U\left(x^{(0)}\right)}{\left(y^{(0)}\right)^{4}}\left(1-\frac{9}{2}\left(y^{(0)}\right)^{-2}\right), \\
& A_{1}^{\prime}=-4 r_{1}+18 r_{1} /\left[2\left(y^{(0)}\right)^{2}-9\right] \\
& A_{2}^{\prime}=10 r_{1}^{2}-4 r_{2}+9\left(2 r_{2}-11 r_{1}^{2}\right) /\left[2\left(y^{(0)}\right)^{2}-9\right], \\
& A_{3}^{\prime}=U^{\prime}\left(x^{(0)}\right) / U\left(x^{(0)}\right), \quad A_{4}^{\prime}=\frac{1}{2} U^{\prime \prime}\left(x^{(0)}\right) / U\left(x^{(0)}\right), \\
& A_{5}^{\prime}=U^{\prime}\left(x^{(0)}\right) / U\left(x^{(0)}\right)\left\{-4 r_{1}+18 r_{1} /\left[2\left(y^{(0)}\right)^{2}-9\right]\right\},(81)
\end{aligned}
$$

а також $t^{(0)}=t_{n}\left(\varphi_{0}, x^{(0)}\right)$. Величини $r_{1}$ і $r_{2}$, які визначаються через функції $U\left(x^{(0)}\right), \varphi\left(x^{(0)}\right)$ та їхні похідні, наведено в $[11,22]$. Для внеску у вільну енергію системи від ділянки КР отримуємо

$$
\begin{aligned}
& F_{\mathrm{CR}}=-k T N^{\prime}\left[a_{0}^{\prime}+\ln Q(d)+\tilde{\gamma}_{01}+\tilde{\gamma}_{02} \tau+\tilde{\gamma}_{03} \tau^{2}-\right. \\
& \left.-\tilde{\gamma}^{\prime} s^{-3\left(m_{\tau}+1\right)}\right] .
\end{aligned}
$$

Коефіцієнти $a_{0}^{\prime}$ і $Q(d)$ означені в (6) та (14). Для інших коефіцієнтів із (82) знаходимо

$$
\begin{aligned}
& \tilde{\gamma}_{01}=s^{-3}\left[\frac{f_{\mathrm{CR}}^{(0)}+\omega_{0}}{1-s^{-3}}+\frac{d_{2} \tilde{c}_{2 k} \tilde{E}_{2}}{1-\tilde{E}_{2} s^{-3}}+\frac{\tilde{d}_{4} \tilde{b}_{0} \tilde{E}_{2}^{2}}{1-\tilde{E}_{2}^{2} s^{-3}}\right] \text {, } \\
& \tilde{\gamma}_{02}=s^{-3}\left[\frac{d_{2} \tilde{c}_{2 k 1} \tilde{E}_{2}}{1-\tilde{E}_{2} s^{-3}}+\frac{\tilde{d}_{4} \tilde{b}_{1} \tilde{E}_{2}^{2}}{1-\tilde{E}_{2}^{2} s^{-3}}+\frac{d_{1} \tilde{c}_{1 k} \tilde{E}_{1}}{1-\tilde{E}_{1} s^{-3}}+\right. \\
& \left.+\frac{\tilde{d}_{5} \tilde{c}_{1 k} \tilde{c}_{2 k} \tilde{E}_{1} \tilde{E}_{2}}{1-\tilde{E}_{1} \tilde{E}_{2} s^{-3}}+\frac{\tilde{d}_{7} \tilde{b}_{0} \tilde{c}_{1 k} \tilde{E}_{1} \tilde{E}_{2}^{2}}{1-\tilde{E}_{1} \tilde{E}_{2} s^{-3}}\right], \\
& \tilde{\gamma}_{03}=s^{-3}\left[\frac{d_{2} \tilde{c}_{2 k 2} \tilde{E}_{2}}{1-\tilde{E}_{2} s^{-3}}+\frac{\tilde{d}_{5}\left(\tilde{c}_{1 k 1} \tilde{c}_{2 k}+\tilde{c}_{1 k} \tilde{c}_{2 k 1}\right) \tilde{E}_{1} \tilde{E}_{2}}{1-\tilde{E}_{1} \tilde{E}_{2} s^{-3}}+\right. \\
& +\frac{\tilde{d}_{4} \tilde{b}_{2} \tilde{E}_{2}^{2}}{1-\tilde{E}_{2}^{2} s^{-3}}+\frac{d_{1} \tilde{c}_{1 k 1} \tilde{E}_{1}}{1-\tilde{E}_{1} s^{-3}}+\frac{\tilde{d}_{3} \tilde{c}_{1 k}^{2} \tilde{E}_{1}^{2}}{1-\tilde{E}_{1}^{2} s^{-3}}+ \\
& +\frac{\tilde{d}_{6} \tilde{c}_{2 k} \tilde{c}_{1 k}^{2} \tilde{E}_{1}^{2} \tilde{E}_{2}}{1-\tilde{E}_{1}^{2} \tilde{E}_{2} s^{-3}}+\frac{\tilde{d}_{7}\left(\tilde{b}_{0} \tilde{c}_{1 k 1}+\tilde{b}_{1} \tilde{c}_{1 k}\right) \tilde{E}_{1} \tilde{E}_{2}^{2}}{1-\tilde{E}_{1} \tilde{E}_{2}^{2} s^{-3}}+ \\
& \left.+\frac{\tilde{d}_{8} \tilde{b}_{0} \tilde{c}_{1 k}^{2} \tilde{E}_{1}^{2} \tilde{E}_{2}^{2}}{1-\tilde{E}_{1}^{2} \tilde{E}_{2}^{2} s^{-3}}\right] \\
& \tilde{\gamma}^{\prime}=\frac{f_{\mathrm{CR}}^{(0)}+\omega_{0}}{1-s^{-3}}+\frac{d_{1} \tilde{f}}{1-\tilde{E}_{1} s^{-3}}+\frac{\tilde{d}_{3} \tilde{f}^{2}}{1-\tilde{E}_{1}^{2} s^{-3}} .
\end{aligned}
$$

Формули (83) відрізняються від аналогічних виразів, одержаних без врахування залежності фур'є-образу потенціалу від хвильового вектора, перенормованими власними значеннями $\tilde{E}_{1}, \tilde{E}_{2}$ і коефіцієнтами $\tilde{d}_{l}$ (iз $l \geq 3$ ), які у випадку врахування згаданої вище залежності мають вигляд

$$
\begin{aligned}
& \tilde{d}_{3}=d_{3}+B_{3}^{2} A_{5}^{\prime \prime} \tilde{E}_{1}^{-1}, \quad \tilde{d}_{4}=d_{4}+B_{1}^{2} A_{5}^{\prime \prime} \tilde{E}_{2}^{-1} \\
& \tilde{d}_{5}=d_{5}+B_{1} B_{3} A_{5}^{\prime \prime}\left(\tilde{E}_{1}^{-1}+\tilde{E}_{2}^{-1}\right) \\
& \tilde{d}_{6}=d_{6}+B_{3} B_{4} A_{5}^{\prime \prime}\left(\tilde{E}_{1}^{-1} \tilde{E}_{2}^{-1}+\tilde{E}_{1}^{-1}\right)+ \\
& +B_{1} B_{6} A_{5}^{\prime \prime}\left(\tilde{E}_{2}^{-1}+\tilde{E}_{1}^{-2}\right) \\
& \tilde{d}_{7}=d_{7}+B_{1} B_{4} A_{5}^{\prime \prime}\left(\tilde{E}_{1}^{-1} \tilde{E}_{2}^{-1}+\tilde{E}_{2}^{-1}\right)+ \\
& +B_{2} B_{3} A_{5}^{\prime \prime}\left(\tilde{E}_{2}^{-2}+\tilde{E}_{1}^{-1}\right)
\end{aligned}
$$




$$
\begin{aligned}
& \tilde{d}_{8}=d_{8}+B_{2} B_{6} A_{5}^{\prime \prime}\left(\tilde{E}_{1}^{-2}+\tilde{E}_{2}^{-2}\right)+ \\
& +B_{3} B_{5} A_{5}^{\prime \prime}\left(\tilde{E}_{1}^{-1}+\tilde{E}_{1}^{-1} \tilde{E}_{2}^{-2}\right)+B_{4}^{2} A_{5}^{\prime \prime} \tilde{E}_{1}^{-1} \tilde{E}_{2}^{-1}+ \\
& +B_{1} B_{7} A_{5}^{\prime \prime}\left(\tilde{E}_{2}^{-1}+\tilde{E}_{1}^{-2} \tilde{E}_{2}^{-1}\right) .
\end{aligned}
$$

Зазначимо, що вирази для $f_{\mathrm{CR}}^{(0)}, d_{l}$ та $B_{l}$ аналогічні наведеним в $[11,19]$. В них замість $A_{l}$ слід підставити $\tilde{A}_{l}=A_{l}+A_{l}^{\prime \prime}$, замість величин $f_{0}$ і $\varphi_{0}$, які характеризують координати фіксованої точки, - нові величини $\tilde{f}$ і $\tilde{\varphi}$, а замість $E_{l}$ та $R_{i j}$ - перенормовані власні значення $\tilde{E}_{l}$ та матричні елементи $\tilde{R}_{i j}$. Це саме стосується і величин $\tilde{c}_{l k m}$ та $\tilde{b}_{l}$, значення яких відрізнятиметься від аналогічних $c_{l k m}$ та $b_{l}$.

Виділяючи в (82) температурну залежність, приходимо до формули

$$
\begin{aligned}
& \text { де } \\
& \tilde{\gamma}_{01}^{\prime}=\bar{a}_{0}+\tilde{\gamma}_{01}, \quad \tilde{\gamma}_{1}=\bar{a}_{1}+\tilde{\gamma}_{02}, \\
& \tilde{\gamma}_{2}=\bar{a}_{2}+\tilde{\gamma}_{03}, \quad \tilde{\gamma}_{10}=\tilde{\gamma}^{\prime} s^{-3 m_{0}} .
\end{aligned}
$$$$
F_{\mathrm{CR}}=-k T N^{\prime}\left[\tilde{\gamma}_{01}^{\prime}+\tilde{\gamma}_{1} \tau+\tilde{\gamma}_{2} \tau^{2}-\tilde{\gamma}_{10} \tau^{3 \nu}\right],
$$

Вирази для $\bar{a}_{l}$ ті ж, що і в $[11,19]$, в яких, однак [22] $x_{0 c}=\sqrt{3}\left[\bar{q}-\tilde{f}+\tilde{c}_{2 k} \tilde{R}^{(0)} \tilde{\varphi}^{-1 / 2}\right] / \sqrt{\tilde{\varphi}+\tilde{c}_{2 k}}$, $\tilde{R}^{(0)}=\tilde{R}_{12}^{(0)} /\left(\tilde{E}_{2}-\tilde{R}_{11}\right)$.

Підкреслимо, що неаналітична частина вільної енергії КР в (85) визначається останнім доданком з перенормованим критичним показником кореляційної довжини

$\nu=\frac{\ln s}{\ln \tilde{E}_{1}}$.

Перенормування цього показника впливає на критичну поведінку теплоємності та інших характеристик системи поблизу температури $T_{c}$.

При розрахунку статистичної суми системи з врахуванням поправки на усереднення потенціалу змінюється функціональний вигляд величини $\tilde{d}_{n+1}(k)$. Для вихідного коефіцієнта $d^{\prime}(k)$ маємо вираз

$a_{2}^{\prime}-\beta \tilde{\Phi}(k)=a_{2}^{\prime}-\beta \tilde{\Phi}(0)+2 b^{2} \beta \tilde{\Phi}(0) k^{2}$.

Після усереднення $k^{2}$ на інтервалі $\left(B_{1}, B^{\prime}\right]$ та інтегрування в нульовому шарі фазового простору K3 коефіцієнт $d^{\prime}(k)$ переходить у величину

$\tilde{d}_{1}(k)=\tilde{a}_{2}^{(1)}-\beta \tilde{\Phi}(0)+2 b^{2} \beta \tilde{\Phi}(0)\left(1+\alpha_{0}\right) k^{2}$.
Середнє значення $\tilde{d}_{1}(k)$ на інтервалі хвильових вектоpiв $\left(B_{2}, B_{1}\right]$ можна подати у вигляді

$\tilde{d}_{1}\left(B_{2}, B_{1}\right)=\tilde{d}_{1}(0)+\bar{q} \beta \tilde{\Phi}(0) s^{-2\left(1-\eta_{0} / 2\right)}$,

де введено позначення

$\eta_{0}=\frac{\alpha_{0}}{\ln s}$.

У термінах середніх значень за рахунок врахування поправки $\Delta \tilde{\Phi}(k)$ виникає перенормування потенціалу взаємодії:

$\left(1+\alpha_{0}\right)\left\langle k^{2}\right\rangle_{B_{2}, B_{1}}=\left\langle k^{2-\eta_{0}}\right\rangle_{B_{2}, B_{1}}$.

Для $n$-ї блочної структури подібним чином отримуєMO

$\tilde{d}_{n}\left(B_{n+1}, B_{n}\right)=\tilde{d}_{n}(0)+2 \beta \tilde{\Phi}(0) b^{2} \times$

$\times\left\langle k^{2}\right\rangle_{B_{n+1}, B_{n}} \prod_{m=0}^{n-1}\left(1+\alpha_{m}\right)$,

що відповідає рівності

$\tilde{d}_{n}\left(B_{n+1}, B_{n}\right)=\tilde{d}_{n}(0)+\bar{q} \beta \tilde{\Phi}(0) \prod_{m=0}^{n-1} s^{-2\left(1-\eta_{m} / 2\right)}$.

Тут

$\eta_{m}=\frac{\alpha_{m}}{\ln s}$

а $\alpha_{m}$ визначене в $(33)$. В області $\mathrm{KP}\left(m \leq m_{\tau}\right)$ виконуються рівності

$\alpha_{0}=\alpha_{1}=\cdots=\alpha_{m}=\alpha^{(0)}$.

Тому величина $\tilde{d}_{n}\left(B_{n+1}, B_{n}\right)$ на ділянці КР може бути представлена у вигляді

$\tilde{d}_{n}\left(B_{n+1}, B_{n}\right)=\tilde{d}_{n}(0)+\bar{q} \beta \tilde{\Phi}(0) s^{-2 n(1-\eta / 2)}$,

де $\eta$ наведено в (70). При цьому будемо мати

$\left(1+\alpha^{(0)}\right)^{n}\left\langle k^{2}\right\rangle_{B_{n+1}, B_{n}}=\left\langle k^{2-\eta}\right\rangle_{B_{n+1}, B_{n}}$.

Якщо температура дорівнює критичній, то

$\lim _{n \rightarrow \infty} \tilde{d}_{n}(0)=0$

і для $\tilde{d}_{n}(k)$ можна записати граничне співвідношення

$\lim _{n \rightarrow \infty} \tilde{d}_{n}(k)=\lim _{k \rightarrow 0} 2 \beta \tilde{\Phi}(0) b^{2} k^{2-\eta}$. 
Розглянемо внесок у вільну енергію системи від довгохвильових флуктуацій. Довжина хвилі, яка відповідає цим флуктуаціям, перевищує кореляційну довжину. Для $T>T_{c}$ у системі, крім критичного, існує ГГР. Він відповідає ділянці хвильових векторів

$0 \leq k<B_{m_{\tau}}$,

де $B_{m_{\tau}}=B^{\prime} s^{-m_{\tau}}$. В області ГГР (або довгохвильових флуктуацій) величина $x_{n}$ (відповідно і $\left.y_{n}\right)$ збільшується з ростом $n$. Це веде до різкого зменшення значень коефіцієнтів $\alpha_{n}$ і функцій $f\left(x_{n}\right), G\left(x_{n}\right), \mathcal{K}\left(x_{n}\right)$. При розрахунку внеску у вільну енергію $F_{\mathrm{LGR}}$ від ділянки ГГР, як і в $[11,19]$, зручно виділити дві області значень хвильового вектора. Перша - перехідна область (ПО), яка відповідає значенням $\mathbf{k}$, близьким до $B_{m_{\tau}}$, тобто шарам фазового простору КЗ безпосередньо після точки виходу із КР. Друга - гаусова область - відноситься до малих значень хвильового вектора $(k \rightarrow 0)$, де розподіл фаз флуктуацій густини спінового моменту набуває гаусового характеру.

Частина вільної енергії системи, що відповідає ПО, має вигляд

$F_{\mathrm{TR}}=-k T N^{\prime} \tilde{f}_{\mathrm{TR}} \tau^{3 \nu}$.

Тут

$\tilde{f}_{\mathrm{TR}}=s^{-3 m_{0}} \sum_{m=0}^{m^{\prime \prime}} s^{-3 m} f_{m_{\tau}+m+1}$,

$f_{m_{\tau}+m+1}=\frac{1}{2} \ln y_{m_{\tau}+m}+\frac{9}{4}\left(y_{m_{\tau}+m}\right)^{-2}+$

$+\frac{x_{m_{\tau}+m+1}^{2}}{4}+\ln U\left(0, x_{m_{\tau}+m+1}\right)$,

а значення $x_{m_{\tau}+m+1}$ i $y_{m_{\tau}+m}$ визначаються згідно 3 формулами

$x_{m_{\tau}+m+1}=\sqrt{3} \frac{\bar{q}+\tilde{f}\left(\tilde{E}_{1}^{m}-1\right)}{\left(\tilde{\varphi}+\tilde{f} \tilde{\varphi}^{1 / 2} \tilde{R}_{1}^{(0)} \tilde{E}_{1}^{m}\right)^{1 / 2}}$,

$y_{m_{\tau}+m}=s^{3 / 2} U\left(x_{m_{\tau}+m}\right)\left(3 / \varphi\left(x_{m_{\tau}+m}\right)\right)^{1 / 2}$.

Величину $m^{\prime \prime}$, яка характеризуе розмір ПО $\left[m_{\tau}+\right.$ $\left.1, m_{\tau}+m^{\prime \prime}+1\right]$, шукаємо відповідно до методики, описаної в $[11,19]$. Ця величина $m^{\prime \prime}$ не залежить від температури і для проміжних значень параметра РГ $s$ становить 2-3 шари фазового простору К3.

Режиму гаусових флуктуацій (гаусова область) відповідає вираз

$$
\begin{aligned}
& Z^{\prime}=2^{-1 / 2}\left(\pi P_{2}^{\left(m_{\tau}^{\prime}-1\right)}\right)^{-\frac{N_{m_{\tau}^{\prime}}}{2}} \times \\
& \times \int \exp \left[-\frac{1}{2} \sum_{k \leq B_{m_{\tau}^{\prime}}} \tilde{d}_{m_{\tau}^{\prime}}(k) \rho_{\mathbf{k}} \rho_{-\mathbf{k}}\right](d \rho)^{N_{m_{\tau}^{\prime}}},
\end{aligned}
$$

де $m_{\tau}^{\prime}=m_{\tau}+m^{\prime \prime}+2$,

$\tilde{d}_{m_{\tau}^{\prime}}(k)=\left(P_{2}^{\left(m_{\tau}^{\prime}-1\right)}\right)^{-1}+\beta\left[\tilde{\Phi}\left(B_{m_{\tau}^{\prime}}, B_{m_{\tau}^{\prime}-1}\right)-\tilde{\Phi}(k)\right]$,

$P_{2}^{\left(m_{\tau}^{\prime}-1\right)}=\left(3 / \tilde{a}_{4}^{\left(m_{\tau}^{\prime}-1\right)}\right)^{1 / 2} U\left(x_{m_{\tau}^{\prime}-1}\right)$.

Беручи до уваги умову $\tilde{c}_{1} \tilde{E}_{1}^{m_{\tau}+1}=\beta \tilde{\Phi}(0) \tilde{f}$, для $\tilde{a}_{4}^{\left(m_{\tau}^{\prime}-1\right)}$ знаходимо

$\tilde{a}_{4}^{\left(m_{\tau}^{\prime}-1\right)}=s^{-2(2-\eta)\left(-\ln \tau / \ln \tilde{E}_{1}+m_{0}\right)} s^{-4 m^{\prime \prime}} \tilde{u}_{m_{\tau}^{\prime}-1}$

або

$\tilde{a}_{4}^{\left(m_{\tau}^{\prime}-1\right)}=\tau^{2(2-\eta) \nu} s^{-2(2-\eta) m_{0}-4 m^{\prime \prime}} \tilde{u}_{m_{\tau}^{\prime}-1}$.

Тут

$\tilde{u}_{m_{\tau}^{\prime}-1}=\tilde{u}+\beta \tilde{\Phi}(0) \tilde{f} \tilde{R}_{1} \tilde{E}_{1}^{m^{\prime \prime}}$.

Тоді згідно з $(101)$ величина $\tilde{d}_{m_{\tau}^{\prime}}(k)$ задовольнятиме співвідношення

$\tilde{d}_{m_{\tau}^{\prime}}(k)=\tau^{2(2-\eta) \nu} s^{-2\left(m_{0}+m^{\prime \prime}\right)+m_{0} \eta} \tilde{G}+$

$+2 \beta \tilde{\Phi}(0) b^{2}\left(1+\alpha^{(0)}\right)^{m_{\tau}+1} k^{2}$,

в якому

$\tilde{G}=\beta \tilde{\Phi}(0) \tilde{g}$,

$\tilde{g}=\left(\frac{\tilde{u}_{m_{\tau}^{\prime}-1}}{3}\right)^{1 / 2} \frac{1}{\beta \tilde{\Phi}(0) U\left(x_{m_{\tau}^{\prime}-1}\right)}-\bar{q}$.

Інтегруючи в (100) за всіма змінними $\rho_{\mathbf{k}} 3 k \neq 0$ та вводячи в розгляд нескінченно мале зовнішне магнітне поле, для складової статистичної суми $Z^{\prime}$ одержуємо

$$
\begin{aligned}
& Z^{\prime}=\left(P_{2}^{\left(m_{\tau}^{\prime}-1\right)}\right)^{-\frac{N_{m_{\tau}^{\prime}}}{2}}(2 \pi)^{-1 / 2} \prod_{k \neq 0}^{B_{m_{\tau}^{\prime}}}\left(\tilde{d}_{m_{\tau}^{\prime}}(k)\right)^{-1 / 2} \times \\
& \times \int \exp \left[-\frac{1}{2} \tilde{d}_{m_{\tau}^{\prime}}(0) \rho_{0}^{2}+\beta \sqrt{N} h \rho_{0}\right] d \rho_{0} .
\end{aligned}
$$

Частина вільної енергії системи, що відповідає $Z^{\prime}$, має вигляд

$$
\begin{aligned}
& F^{\prime}=\frac{1}{2} k T\left[N_{m_{\tau}^{\prime}} \ln P_{2}^{\left(m_{\tau}^{\prime}-1\right)}+\sum_{k=0}^{B_{m_{\tau}^{\prime}}} \ln \tilde{d}_{m_{\tau}^{\prime}}(k)\right]- \\
& -N \frac{s^{2\left(m_{0}+m^{\prime \prime}\right) s^{-m_{0} \eta}}}{2 \tilde{\Phi}(0) \tilde{g}} h^{2} \tau^{-(2-\eta) \nu} .
\end{aligned}
$$

У результаті врахування (101), (104) та підсумовування в (107) за хвильовими векторами приходимо до формули

$F^{\prime}=-k T N^{\prime} \tilde{f}^{\prime \prime} \tau^{3 \nu}-\beta N \tilde{\gamma}_{4}^{+} h^{2} \tau^{-(2-\eta) \nu}$, 
де

$\tilde{f}^{\prime \prime}=\tilde{f}^{\prime} \tilde{c}_{\nu}^{3} s^{-3\left(m^{\prime \prime}+1\right)}$,

$\tilde{\gamma}_{4}^{+}=\tilde{c}_{\nu}^{-(2-\eta)} \bar{\gamma}_{4}^{+} /(\beta \tilde{\Phi}(0)), \quad \bar{\gamma}_{4}^{+}=s^{2 m^{\prime \prime}} /(2 \tilde{g})$,

$\tilde{f}^{\prime}=-\frac{1}{4} \ln 3+\frac{1}{3}+\frac{1}{4} \ln \left(\tilde{\varphi}+\tilde{f} \tilde{\varphi}^{1 / 2} \tilde{E}_{1}^{m^{\prime \prime}} \tilde{R}_{1}^{(0)}\right)-$

$-\frac{1}{2} \ln \left(s^{-2}+\tilde{g}\right)-\tilde{g}^{\prime}\left(1-\sqrt{\tilde{g}^{\prime}} \operatorname{arctg} \frac{1}{\sqrt{\tilde{g}^{\prime}}}\right)-$

$-\frac{1}{2} \ln U\left(x_{m_{\tau}^{\prime}-1}\right)$,

$\tilde{c}_{\nu}=\left(\tilde{c}_{1 k} / \tilde{f}\right)^{\nu}, \quad \tilde{R}_{1}^{(0)}=\left(\tilde{E}_{1}-\tilde{R}_{11}\right) / \tilde{R}_{12}^{(0)}, \quad \tilde{g}^{\prime}=\tilde{g} s^{2}$.

Відзначимо, що $s^{-m_{0}}=\tilde{c}_{\nu}$.

Розраховані внески у вільну енергію системи $F_{\mathrm{CR}}$ (див. (85)) та $F_{\mathrm{LGR}}=F_{\mathrm{TR}}+F^{\prime}$ (див. $(97),(108)$ ) дають змогу відповідно до (75) записати повний вираз для неї. Будемо мати

$F=-k T N^{\prime}\left[\tilde{\gamma}_{0}+\tilde{\gamma}_{1} \tau+\tilde{\gamma}_{2} \tau^{2}+\tilde{\gamma}_{3} \tau^{3 \nu}\right]-$

$-\beta N \tilde{\gamma}_{4}^{+} h^{2} \tau^{-(2-\eta) \nu}$.

Тут

$\tilde{\gamma}_{0}=\tilde{\gamma}_{01}^{\prime}+s_{0}^{3} \ln 2$,

$\tilde{\gamma}_{3}=-\tilde{\gamma}_{10}+\tilde{f}_{\mathrm{TR}}+\tilde{f}^{\prime \prime}$,

коефіцієнти $\tilde{\gamma}_{1}, \tilde{\gamma}_{2}$ означено в (86), а $\tilde{\gamma}_{4}^{+}-$в (109). Ентропія та теплоємність системи можуть бути знайдені із виразу для вільної енергії (110) шляхом прямого диференціювання за температурою при $h=0$.

Сприйнятливість системи на одну частинку отримаємо, обчисливши другу похідну вільної енергії за зовнішнім полем $\mathcal{H}$ :

$\chi=\tilde{\Gamma}^{+} \tau^{-\gamma} \frac{\mu_{\mathrm{B}}^{2}}{\tilde{\Phi}(0)}$.

Критичний показник сприйнятливості задається виразом

$\gamma=(2-\eta) \nu$

Величина

$\tilde{\Gamma}^{+}=2 \tilde{c}_{\nu}^{-(2-\eta)} \bar{\gamma}_{4}^{+}$

є критичною амплітудою.

Т а б ли ц я 2. Значення критичних показників з врахуванням поправки на усереднення фур'є-образу потенціалу $(\Delta \tilde{\Phi}(k) \neq 0)$ і без неї $(\Delta \tilde{\Phi}(k)=0)$

\begin{tabular}{c|c|c|c|c}
\hline Умова & $\eta$ & $\nu$ & $\gamma$ & $\alpha$ \\
\hline$\Delta \tilde{\Phi}(k)=0$ & 0 & 0,612 & 1,225 & 0,163 \\
$\Delta \tilde{\Phi}(k) \neq 0$ & 0,024 & 0,577 & 1,141 & 0,268 \\
\hline
\end{tabular}

Критичні показники кореляційної функції $\eta$, кореляційної довжини $\nu$, сприйнятливості $\gamma$ та теплоємності $\alpha=2-3 \nu$, обчислені в наближенні четвірної густини міри для параметра РГ $s=4$ при $\Delta \tilde{\Phi}(k)=0$ і в лінійному наближенні за $\Delta \tilde{\Phi}(k)$, наведено в табл. 2.

Зазначимо, що знайдені критичні показники, зокрема критичний показник кореляційної функції $\eta=0,024$, узгоджуються з даними інших авторів. Так, наприклад, оцінки $\eta=0,0335(25), \eta=0,0362(8)$ i $\eta=0,033$ були одержані відповідно в рамках теоретико-польового підходу (7-ми петлеві розрахунки) [27], Монте-Карло симуляцій [28] і непертурбативного РГ підходу (порядок $\partial^{4}$ розкладу за похідними) $[14]$.

\section{6. Висновки}

Відомі теоретичні підходи, присвячені вирішенню проблеми фазових переходів та критичних явищ (див., наприклад, [20, 29-35]), грунтуються переважно на використанні гаусової густини міри як базисної. За такого підходу розрахунок вільної енергії та інших термодинамічних функцій системи здійснюється з врахуванням залежності фур'є-образу потенціалу $\tilde{\Phi}(k)$ від хвильового вектора. Проте, використання гаусової густини міри як базисної передбачає застосування теорії збурень. Застосування в критичній області методів традиційної теорії збурень з гаусовим розподілом приводить до виникнення безмежної множини розбіжних при $T \rightarrow T_{c}$ діаграм для вільної енергії. Внаслідок цього, при розрахунку статистичної суми виникає альтернатива: або користуватися теорією збурень і враховувати залежність фур'єобразу потенціалу взаємодії від хвильового вектора k, або, усереднюючи (на певних інтервалах $k$ ) фур'єобраз потенціалу взаємодії, використовувати негаусовий розподіл флуктуацій (загалом довільної складності) як базисний. Останнє дає змогу уникнути проблеми виникнення нефізичних розбіжностей при розрахунку вільної енергії поблизу $T_{c}$ і отримати не лише правильні якісні, а й кількісні результати. Однак у низці робіт (див., наприклад, [15-18]) в рамках такого підходу використовувалося наближення, що приводило до нульового значення критичного показника кореляційної функції $(\eta=0)$. Викладена в даній роботі методика досліджень дозволяє не лише отримати відмінний від нуля критичний показник $\eta$, а й знайти більш загальні температурні залежності термодинамічних характеристик тривимірної системи поблизу точки фазового переходу. 
У статті розвинуто метод розрахунку вільної енергії тривимірної ізингоподібної системи у випадку використання четвірної густини міри як базисної (моделі $\rho^{4}$ ), коли береться до уваги залежність фур'єобразу потенціалу взаємодії від хвильового вектора. Поправка на усереднення потенціалу $\tilde{\Phi}(k)$ враховується в лінійному наближенні. При цьому записано і досліджено РС між коефіцієнтами ефективних густин мір. Показано, що врахування поправки на усереднення потенціалу приводить до зміни асимптотики розв'язків $\mathrm{PC}$ при $T=T_{c}$. Розроблено аналітичний спосіб розрахунку малого критичного показника кореляційної функції, який виникає через поправку, та знайдено його значення.

Врахування поправки на усереднення потенціалу веде до перенормування критичних показників кореляційної довжини, сприйнятливості, теплоємності системи. Змінюються також критичні амплітуди. Перенормування критичного показника кореляційної довжини $\nu$ (порівняно з випадком $\eta=0$ ) пов'язане зі зміною більшого власного значення матриці лінійного перетворення РГ. На відміну від $\nu$, критичний показник сприйнятливості $\gamma$ явно залежить від $\eta$ і визначається відповідно до (113). Теплоємність системи характеризується показником $\alpha$, вираз для якого містить перенормований критичний показник кореляційної довжини $\nu$.

Як бачимо із даних табл. 2, врахування ненульового показника $\eta$ в методі К3 приводить до зменшення значення критичного показника кореляційної довжини $\nu$ (як це має місце в непертурбативному РГ підході [14]). Для одержання кращих кількісних оцінок цього та інших критичних показників необхідно враховувати складніші від четвірного розподіли флуктуацій (наприклад, шестирний), в рамках яких показник $\nu$ без врахування поправки на усереднення потенціалу $(\eta=0)$ набуває більших значень, ніж у випадку моделі $\rho^{4}[11]$.

Розвинуту методику досліджень та отримані результати можна використати під час вивчення властивостей різноманітних систем, зокрема феромагнетиків, антиферомагнетиків, сегнетоелектриків, системи рідина-газ, бінарних сумішей, граткової моделі рідини.

1. K.G. Wilson, Phys. Rev. B 4, 3174 (1971); 4, 3184 (1971).

2. K.G. Wilson and M.E. Fisher, Phys. Rev. Lett. 28, 240 (1972).

3. L.P. Kadanoff, Physics 2, 263 (1966).

4. А.З. Паташинский, В.Л. Покровский, ЖЭТФ 50, 439 (1966).
5. И.Р. Юхновский, ДАН СССР 232, 312 (1977).

6. И.Р. Юхновский, Ю.К. Рудавский, ДАН СССР 233, 579 (1977).

7. И.Р. Юхновский, ТМФ 36, 373 (1978).

8. И.Р. Юхновский, Фазовые переходы второго рода. Метод коллективных переменных (Наукова думка, Киeв, 1985).

9. I.R. Yukhnovskii, Phase Transitions of the Second Order. Collective Variables Method (World Scientific, Singapore, 1987).

10. I.R. Yukhnovskii, Riv. Nuovo Cimento 12, 1 (1989).

11. I.Р. Юхновський, М.П. Козловський, І.В. Пилюк, $M i$ кроскопічна теорія фазових переходів у тривимірних системах (Євросвіт, Львів, 2001).

12. І.Р. Юхновський, Вибрані праці. Фізика (Видавництво Національного університету “Львівська політехніка", Львів, 2005).

13. J. Berges, N. Tetradis, and C. Wetterich, Phys. Rep. 363, 223 (2002).

14. L. Canet, B. Delamotte, D. Mouhanna, and J. Vidal, Phys. Rev. B 68, 064421 (2003).

15. И.В. Пылюк, М.П. Козловский, Изв. АН СССР. Сер. физич. 55, 597 (1991).

16. I.R. Yukhnovskii, M.P. Kozlovskii, and I.V. Pylyuk, Phys. Rev. B 66, 134410 (2002).

17. M.P. Kozlovskii, I.V. Pylyuk, and O.O. Prytula, Nucl. Phys. B 753[FS], 242 (2006).

18. I.V. Pylyuk and M.P. Kozlovskii, Physica A 389, 5390 (2010).

19. M.P. Kozlovsky and I.V. Pylyuk, Preprint ITP-85-23E (Bogolyubov Institute for Theor. Physics, Kiev, 1985).

20. К. Вильсон, Дж. Когут, Ренормализачионная группа и є-разложение (Мир, Москва, 1975).

21. M.P. Kozlovskii and Ja.N. Ilnytskii, Preprint ITP-87100E (Bogolyubov Institute for Theor. Physics, Kiev, 1987).

22. И.Р. Юхновский, М.П. Козловский, И.В. Пылюк, Препринт Института теоретической физики, ИТФ-88105Р (ИТФ, Киев, 1988).

23. И.Р. Юхновский, В.М. Ткачук, Препринт Института теоретической физики, ИТФ-81-70Р (ИТФ, Киев, 1981).

24. И.Р.Юхновский, Ю.К. Рудавский, Ю.В. Головач, Препринт Института теоретической физики, ИТФ-82-130Р (ИТФ, Киев, 1982).

25. I.R. Yukhnovskii, M.P. Kozlovskii, and I.V. Pylyuk, Z. Naturforsch. 46a, 1 (1991).

26. М.П. Козловский, ДАН УССР. Сер. А №10, 58 (1984).

27. R. Guida and J. Zinn-Justin, J. Phys. A 31, 8103 (1998).

28. M. Hasenbusch, Int. J. Mod. Phys. C 12, 911 (2001). 
29. М. Фишер, Природа критического состояния (Мир, Москва, 1968).

30. Г. Стенли, Фазовые переходы и критические явления (Мир, Москва, 1973).

31. Ш. Ма, Современная теория критических явлений (Мир, Москва, 1980).

32. А.З. Паташинский, В.Л. Покровский, Флуктуационная теория фазовых переходов (Наука, Москва, 1982).

33. D.J. Amit, Field Theory, the Renormalization Group, and Critical Phenomena (World Scientific, Singapore, 1984).

34. J. Zinn-Justin, Quantum Field Theory and Critical Phenomena (Clarendon Press, Oxford, 1996).

35. H. Kleinert and V. Schulte-Frohlinde, Critical Properties of $\varphi^{4}$-Theories (World Scientific, Singapore, 2001).

Одержано 07.09.11

\section{МЕТОД РАСЧЕТА}

СВОБОДНОЙ ЭНЕРГИИ ТРЕХМЕРНОЙ

ИЗИНГОПОДОБНОЙ СИСТЕМЫ С УЧЕТОМ ПОПРАВКИ

НА УСРЕДНЕНИЕ ПОТЕНЦИАЛА ВЗАИМОДЕЙСТВИЯ

И.Р. Юхновский, М.П. Козловский, И.В. Пълюк

$\mathrm{P}$ е $з$ ю м е

Работа посвящена теоретическому изучению критического поведения трехмерных модельных систем. Выполнен расчет статистической суммы и свободной энергии однокомпонентной спиновой системы с использованием негауссова распределения флуктуаций параметра порядка. Особенностью предложенного метода расчета является учет зависимости фурье-образа потенциала взаимодействия от волнового вектора. Это приводит к отличному от нуля критическому показателю корреляцион- ной функции $\eta$ и перенормированию значений других критических показателей (корреляционной длины, восприимчивости и т.п.). Расчет последних осуществлен с использованием метода ренормализационной группы на основе полученных в работе рекуррентных соотношений между коэффициентами распределений флуктуаций в смежных блочных структурах.

\section{METHOD FOR THE CALCULATION OF FREE \\ ENERGY IN A THREE-DIMENSIONAL ISING-LIKE SYSTEM TAKING INTO ACCOUNT A CORRECTION FOR THE INTERACTION POTENTIAL AVERAGING}

\section{I.R. Yukhnovskii, M.P. Kozlovskii, I.V. Pylyuk}

Institute for Condensed Matter Physics, Nat. Acad. of Sci. of Ukraine

(1, Svientsitskii Str., Lviv 79011, Ukraine; e-mail:piv@icmp.lviv.ua)

S u m m a r y

The critical behavior of three-dimensional model systems has been studied theoretically. The partition function and the free energy for a one-component spin system have been calculated for a nonGaussian distribution of order-parameter fluctuations. A specific feature of the proposed method of calculation consists in making allowance for the dependence of the Fourier transform of the interaction potential on the wave vector. Such an approach leads to a nonzero critical exponent $\eta$ in the correlation function and the renormalization of the values of other critical exponents (for the correlation length, susceptibility, etc.). The calculation of those exponents was carried out with the use of the renormalizationgroup method and on the basis of obtained recurrence relations for the coefficients of fluctuation distributions in adjacent block structures. 